\title{
GEOMETRY OF SECOND ADJOINTNESS FOR $p$-ADIC GROUPS
}

\author{
ROMAN BEZRUKAVNIKOV AND DAVID KAZHDAN, \\ WITH AN APPENDIX BY YAKOV VARSHAVSKY, ROMAN BEZRUKAVNIKOV, \\ AND DAVID KAZHDAN \\ Dedicated to the memory of Izrail' Moiseevich Gel'fand
}

\begin{abstract}
We present a geometric proof of second adjointness for a reductive $p$-adic group. Our approach is based on geometry of the wonderful compactification and related varieties. Considering asymptotic behavior of a function on the group in a neighborhood of a boundary stratum of the compactification, we get a "cospecialization" map between spaces of functions on various varieties carrying a $G \times G$ action. These maps can be viewed as maps of bimodules for the Hecke algebra, and the corresponding natural transformations of endo-functors of the module category lead to the second adjointness. We also get a formula for the "cospecialization" map expressing it as a composition of the orispheric transform and inverse intertwining operator; a parallel result for $D$-modules was obtained by Bezrukavnikov, Finkelberg and Ostrik. As a byproduct we obtain a formula for the Plancherel functional restricted to a certain commutative subalgebra in the Hecke algebra generalizing a result by Opdam.
\end{abstract}

\section{Contents}

1. Introduction

2. Algebraic varieties related to a semi-simple group

2.1. Standard notations

2.2. The spaces $X, Y$

2.3. Partial compactifications of $Y$

2.4. The wonderful compactification

2.5. More on partial compactifications of $X$

3. Co-specializaton from normal bundle for functions on a $p$-adic manifold

3.1. The almost homogeneous case

3.2. The singular case

4. An algebraic lemma

5. Construction and properties of the maps $\mathrm{B}_{I}$

5.1. Definition of $\mathrm{B}_{I}$

5.2. The induced map on $U_{P}$ coinvariants

5.3. The map $\mathrm{B}_{I}$ and $K_{0}$ cosets

6. Second adjointness

Received by the editors April 2, 2014 and, in revised form, September 3, 2015 and October 4, 2015 .

2010 Mathematics Subject Classification. Primary 20G05, 20G25, 22E35, 22E50.

R.B. was supported by the NSF grant DMS-1102434 and a Simons Foundation fellowship.

D.K. was supported by the ERC grant 669655 and US-Israel Binational Science Foundation grant 2012365. 
6.1. Basic notations

6.2. The adjunction maps

6.3. Second adjointness

7. Bernstein's map and intertwining operators 318

7.1. Bounded supports

7.2. Intertwining operators

7.3. Main result

8. Some properties of Radon correspondence

8.1. Proof of Propositions 7.3, 7.5

8.2. The Bernstein center and supports

8.3. Completion of the proof of Proposition 7.1

9. Proof of Theorem 7.6 323

9.1. The subquotient maps for $\mathrm{B}^{\star} \quad 323$

9.2. Proof of Theorem 7.6

10. Plancherel type formulas 325

10.1. The map B and the Plancherel functional 325

10.2. Commutative subalgebras in the Hecke algebra 326

10.3. Proof of Proposition 7.2

10.4. Plancherel functional on the abelian subalgebra 328

11. Appendix: quasi-normal cone for toric coverings 329

11.1. Quasi-normal cone 330

11.2. Toric covering 330

11.3. Theorem 331

Acknowledgements 331

References

\section{Introduction}

Parabolic induction and restriction (Jacquet) functors play a fundamental role in representation theory of reductive $p$-adic groups. It follows directly from definitions that the parabolic induction functor is right adjoint to the Jacquet functor; we will call this adjointness the ordinary or Frobenius adjointness. It has been discovered by Casselman (cf. [8, Theorem 4.2.4]) for admissible representations and generalized by Bernstein to arbitrary smooth representations that there is another nonobvious adjointness between the two functors. Namely, the parabolic induction functor turns out to be also left adjoint to Jacquet functor with respect to the opposite parabolic (we will refer to this as the second or the Bernstein adjointness). This fact appears in unpublished notes of Bernstein [1] (see also exposition in 16]), we reprove it below. Rather than following the original strategy, our approach emphasizes the relation to geometry of the group and related spaces. More precisely, showing that the two functors $\alpha$ and $\beta$ are adjoint amounts to providing morphisms between the identity functor and the compositions $\alpha \circ \beta, \beta \circ \alpha$ satisfying certain compatibilities. When $\alpha, \beta$ are Jacquet and parabolic induction functors, the arrow between the endo-functors of the category of representations of the Levi is easy to define, both for the ordinary and for the second adjointness. To describe the morphisms between the endo-functors of representations of $G$, recall that an endo-functor of a category of modules usually comes from a bimodule. This is so in our case, moreover, the 
bimodules can be realized as spaces of functions 1 on a variety equipped with a $G \times G$-action. Thus showing adjointness amounts to providing a map between the function spaces satisfying certain properties. For the ordinary induction, this turns out to be the map from functions on the group to integral kernels acting on the universal principal series, i.e., on the space of functions on $G / U$. This well-studied map (known as the orispheric transform) is given by an explicit correspondence.

In contrast, the map responsible for the second adjointness has not, to our knowledge, received much attention in the literature 2 The goal of this paper is a geometric description of this map and its basic properties. It is a map (to be denoted by B) from functions on (the space of $F$-points of) a certain algebraic variety $X$ to the space of functions on $G$. Our first observation is that $X$ is an asymptotic cone of $G$. By this we mean that it admits an open embedding to the normal space $N_{\bar{G}}(Z)$ where $\bar{G}$ is the De Concini-Procesi compactification of $G$ and $Z \subset \bar{G}$ is a $G \times G$ orbit 3 The map $B$ is uniquely characterized by the property of being asymptotic to identity and $G \times G$-equivariant; see Corollary 5.2 (this is also an outgrowth of an idea communicated to us by Bernstein).

The second main result of the paper addresses the question of presenting this map by an explicit correspondence. The answer is that the correspondence giving B can be expressed in terms of the inverse to the intertwining operator (Radon transform); see Theorem 7.6. As an application of this result we obtain a generalization of a result of Opdam which describes the Plancherel functional restricted to a certain commutative subalgebra in the Hecke algebra.

This paper is structured as follows. In section 2 we collect necessary algebrogeometric facts about the wonderful compactification and related varieties. Section 3 develops the formalism of transferring functions between a $p$-adic variety and the normal bundle to its subvariety. Section 4 contains some algebraic preliminaries. Those sections are mutually logically independent. In section 5 the results of the earlier sections are combined to get the desired map B. In section [ second adjointness is deduced. In section 7 we formulate some properties of the map B, including the formula expressing it in terms of the inverse to the intertwining operator. In sections 8 and 9 we prove some statements stated in section 7 . Finally, section 10 contains a generalization of Opdam's formula. In the Appendix (joint with Y. Varshavsky) we describe a version of the normal bundle construction which allows us to extend some statements about De Concini-Procesi's wonderful compactification to reductive groups with a nontrivial center.

We did not attempt to reach a maximal reasonable generality. In particular, we expect that the present methods can be used to obtain a generalization of some of our results (such as Theorem (7.6) replacing a Borel subgroup by an arbitrary parabolic.

\footnotetext{
${ }^{1}$ Here and elsewhere in the Introduction we ignore the difference between functions and measures; see the precise statement in section 6

${ }^{2}$ See, however, [17] (which appeared after the preprint version of the present paper) where related ideas have been developed and applied in a more general context.

${ }^{3}$ When the group $G$ is not of adjoint type the compactification is not smooth. In this case we use a modification of the notion of normal cone introduced in the Appendix.
} 


\section{Algebraic varieties related to a Semi-Simple group}

In this section we collect algebro-geometric facts to be used in the main construction. Until the end of the section we work over an arbitrary field which we denote by $F$. In this section topological notions refer to Zarisky topology.

2.1. Standard notations. Let $G$ be a connected split reductive algebraic group over $F$. Let $\mathcal{B}$ be the space of Borel subgroups of $G$ and $W$ be the (abstract) Weyl group. Let $\mathbb{T}$ be the abstract Cartan of $G$, thus $\mathbb{T}$ is canonically identified with $B / U_{B}$ for any $B \in \mathcal{B}$, where $U_{B}$ is the unipotent radical of $B$. The torus $\mathbb{T}$ acts on the right on $G / U_{B}$ for any $B \in \mathcal{B}$.

We denote by $X_{*}(\mathbb{T})$ the lattice of cocharacters of the torus $\mathbb{T}$ and by $X^{*}(\mathbb{T})$ its lattice of characters. By $X_{*}(\mathbb{T})^{+}, X^{*}(\mathbb{T})^{+}$we denote the subsets of dominant (co)characters. Let $\Sigma$ be the set of vertices of the Dynkin diagram of $G$. We use the bijection between conjugacy classes of parabolic subgroups in $G$ and subsets in $\Sigma$ sending a parabolic to the set of $i \in \Sigma$ such that the corresponding root subspace is in the radical of $P$. For $I \subset \Sigma$ we let $P_{I}=L_{I} U_{I}$ denote (an arbitrary) representative of the corresponding conjugacy class.

For $i \in \Sigma$ we denote by $\alpha_{i}$ the corresponding simple root, by $\alpha_{i}^{\vee}$ the corresponding simple coroot and by $\omega_{i}$ the corresponding fundamental weight. The abstract Weyl group $W$ acts on $\mathbb{T}$, for $i \in \Sigma$ denote by $s_{i} \in W$ the corresponding simple reflection and let $w_{0}$ denote the longest element of $W$. For a pair $B, B^{\prime}$ of Borel subgroups we denote by $w\left(B, B^{\prime}\right) \in W$ their relative position, with an alternative notation $B \stackrel{w}{-} B^{\prime}$. We often fix a Borel subgroup $B_{0} \in \mathcal{B}$ and denote by $\mathcal{B}_{w}$ the Schubert cell given by $\mathcal{B}_{w}=\left\{B \in \mathcal{B} \mid B_{0} \stackrel{w}{-} B\right\}$.

2.2. The spaces $X, Y$. Fix $B_{1}, B_{2} \in \mathcal{B}$ and set $X_{B_{1}, B_{2}}=\left(G / U_{B_{1}} \times G / U_{B_{2}}\right) /\left(B_{1} \cap\right.$ $B_{2}$ ), where $B_{1} \cap B_{2}$ acts diagonally on the right. Given another choice $\left(B_{1}^{\prime}, B_{2}^{\prime}\right) \in \mathcal{B}^{2}$ in the same conjugacy class, an element $g \in G$ such that $B_{i}^{\prime}=g B_{i} g^{-1}$ for $i=1,2$ is defined uniquely up to right multiplication by an element of $B_{1} \cap B_{2}$. Then the map $y \mapsto y g^{-1}$ induces isomorphisms $G / U_{B_{i}} \simeq G / U_{B_{i}^{\prime}}$. The induced isomorphism $X_{B_{1}, B_{2}} \simeq X_{B_{1}^{\prime}, B_{2}^{\prime}}$ does not depend on the choice of $g$, thus the conjugacy class of the pair $\left(B_{1}, B_{2}\right)$ defines $X_{B_{1}, B_{2}}$ uniquely up to a unique isomorphism. Such conjugacy classes are in bijection with $W$, thus for each $w \in W$ we get a welldefined variety $X_{w}=X_{B_{1}, B_{2}}$, where $w=w\left(B_{1}, B_{2}\right)$. By construction the group $\mathbb{T}^{2}$ acts on varieties $X_{w}$.

We will only use two extreme cases for which we fix a different notation: $X=X_{w_{0}}$ and $Y=X_{e}$ (where $e$ is the unit element). Fixing $B \in \mathcal{B}$ we get $X=\left(G / U_{B}\right)^{2} / \mathbb{T}$, $Y=\left(G / U_{B}\right)^{2} / \mathbb{T}$ where the torus acts via the maps $\mathbb{T} \rightarrow \mathbb{T}^{2}$ given by $t \mapsto\left(t, w_{0}(t)\right)$ and $t \mapsto(t, t)$ respectively.

We denote by $p_{X}: X \rightarrow \mathcal{B}^{2}, p_{Y}: Y \rightarrow \mathcal{B}^{2}$ the natural projections.

Notice that the stabilizer in $G$ of the unit coset $\left(U_{B_{1}}, U_{B_{2}}\right) \bmod \left(B_{1} \cap B_{2}\right)$ coincides with the stabilizer of its image in $\mathcal{B}^{2}$. It follows in particular that

$$
\begin{aligned}
& p_{X}^{-1}\left(\mathcal{B}_{0}^{2}\right) \cong \mathcal{B}_{0}^{2} \times \mathbb{T}^{2} / \mathbb{T}_{\Delta} \cong \mathcal{B}_{0}^{2} \times \mathbb{T}, \\
& p_{Y}^{-1}\left(\Delta_{\mathcal{B}}\right) \cong \Delta_{\mathcal{B}} \times \mathbb{T}^{2} / \mathbb{T}_{\Delta} \cong \mathcal{B} \times \mathbb{T},
\end{aligned}
$$

where $\Delta_{\mathcal{B}} \subset \mathcal{B}^{2}$ is the diagonal, $\mathcal{B}_{0}^{2} \subset \mathcal{B}^{2}$ is the open orbit of the diagonal $G$-action, and $\mathbb{T}_{\Delta} \subset \mathbb{T}^{2}$ is the diagonal subtorus. The isomorphisms $\mathbb{T}^{2} / \mathbb{T}_{\Delta} \cong \mathbb{T}$ are given by $\left(t_{1}, t_{2}\right) \mapsto t_{1}^{-1} t_{2}$. 
Example 2.1. Let $G=S L(2)$. Then $Y$ parametrizes pairs of nonzero vectors $v_{1}, v_{2} \in F^{2}$ modulo common dilations, while $X$ is the space of rank one $2 \times 2$ matrices (notice that the symplectic form on the two-dimensional space identifies it with its dual). Also, $p_{Y}^{-1}\left(\Delta_{\mathcal{B}}\right)$ parametrizes pairs of nonzero colinear vectors, the map $p_{Y}^{-1}\left(\Delta_{\mathcal{B}}\right) \rightarrow \mathbb{T}=\mathbb{G}_{m}$ sends such a pair $(v, \lambda v)$ to $\lambda$. The space $p_{X}^{-1}\left(\mathcal{B}_{0}^{2}\right)$ parametrizes nonnilpotent rank one matrices, the map $p_{X}^{-1}\left(\mathcal{B}_{0}^{2}\right) \rightarrow \mathbb{T}$ sends such a matrix to its trace.

2.2.1. The space $X_{I}$. The definition of $X$ can be generalized as follows. Given two opposite parabolic subgroups $P, P^{-}$with unipotent radicals $U_{P}, U_{P^{-}}$, an argument similar to the one above shows that the space $\left(G / U_{P} \times G / U_{P^{-}}\right) /\left(P \cap P^{-}\right)$depends only on the conjugacy class of $P$. Thus we get a variety defined uniquely up to a unique isomorphism for every conjugacy class of parabolic subgroups. The latter are in a bijection with subsets $I \subset \Sigma$ (see 2.1), for such a subset $I$ we let $X_{I} \cong\left(G / U_{P_{I}} \times G / U_{P_{I}^{-}}\right) /\left(P_{I} \cap P_{I}^{-}\right)$denote the corresponding variety. Thus $X=X_{\Sigma}$.

Similarly, set $Y_{I}=\left(G / U_{P_{I}} \times G / U_{P_{I}}\right) / L_{I}$.

Remark 2.2. The algebraic variety $X_{I}$ has been defined using a choice of the parabolic subgroup $P_{I}$; however, this variety (like $G / P$ but unlike $G / U_{P}$ or $Y_{I}$ ) is defined canonically given the reductive group $G$. This is clear in view of the following: given two pairs of opposite parabolics $\left(P_{I}, P_{I}^{-}\right)$and $\left(P_{I}^{\prime},\left(P_{I}^{-}\right)^{\prime}\right)$ in the same conjugacy class, an element conjugating $\left(P_{I}^{\prime},\left(P_{I}^{-}\right)^{\prime}\right)$ to $\left(P_{I}, P_{I}^{-}\right)$is unique up to multiplication by an element in $L$, thus the isomorphism between the corresponding quotients is canonical.

\subsubsection{Radon correspondence.}

Lemma 2.3. For any $w \in W$ there exists unique orbit $\mathfrak{C}_{w}$ of the diagonal $G^{2}$ action on $X \times Y$, such that:

i) For any $\left(x_{1}, x_{2}, y_{1}, y_{2}\right) \in \mathcal{B}^{4}$ in the image of $\mathfrak{C}_{w}$ under $p_{X} \times p_{Y}$ we have $x_{1} \stackrel{w}{-} y_{1}$, ${ }_{2}-y_{2}$

ii) For any $x \in p_{X}^{-1}\left(\mathcal{B}_{0}^{2}\right), y \in p_{Y}^{-1}\left(\Delta_{\mathcal{B}}\right)$ such that $x, y$ go to the unit element in $\mathbb{T}$ under the projection to the second factor in the decompositions (2.1), (2.2), we have $(x, y) \in \mathfrak{C}_{w}$.

Proof. It is clear that for any $x \in X$ orbits of $G^{2}$ on $X \times Y$ are in bijection with orbits of the stabilizer $\operatorname{Stab}_{G \times G}(x)$ on $Y$. If $x \in p_{X}^{-1}\left(\Delta_{\mathcal{B}}\right)$, then $\operatorname{Stab}_{G \times G}(x)=$ $\mathbb{T} \cdot\left(U_{B_{0}} \times U_{B_{0}}\right)$ for some Borel subgroup $B_{0}=\mathbb{T} \cdot U_{B_{0}}$. Thus $\operatorname{Stab}_{G \times G}(x)$ has a unique orbit in $p_{Y}^{-1}\left(\mathcal{B}_{w} \times \mathcal{B}_{w w_{0}}\right)$ containing a point $y$ which goes to the unit element in $\mathbb{T}$ under the projection to the second factor in (2.2). It is clear that this orbit is independent of the choice of $x \in p_{X}^{-1}\left(\Delta_{\mathcal{B}}\right)$ provided that $x$ goes to the unit element in $\mathbb{T}$ under the projection to the second factor in (2.1).

We call $\mathfrak{C}_{w}$ the Radon correspondence.

Example 2.4. Let $G=S L(2)$, the spaces $X$ and $Y$ are described in Example 2.1. Then $\mathfrak{C}_{e}=\left\{\left(m ; v_{1}, v_{2}\right) \in X \times Y \mid m\left(v_{2}\right)=v_{1}\right\}$ and $\mathfrak{C}_{s}=\left\{\left(m ; v_{1}, v_{2}\right) \in\right.$ $\left.X \times Y \mid m^{t}\left(v_{1}\right)=v_{2}\right\}$ where $m^{t}$ is the transposed matrix with respect to the symplectic form on the two-dimensional vector space and $e, s$ are the two elements in $W \cong \mathbb{Z} / 2 \mathbb{Z}$. 
2.2.3. Radon correspondence is closed. Variety $X$ is well known to be quasi-affine (see e.g. [19, Exercise 5.5.9(2)]), let $\bar{X}_{\text {aff }}$ be its affine closure.

Lemma 2.5. The image of $\mathfrak{C}_{w}$ under the open embedding $X \times Y \rightarrow \bar{X}_{\text {aff }} \times Y$ is closed.

Proof. Since the subset $\mathfrak{C}_{w} \subset \bar{X}_{\text {aff }} \times Y$ is $G \times G$-invariant and $G \times G$ acts transitively on $Y$, it is sufficient to show that fibers $\mathfrak{C}_{w}(y)$ of the projection $\mathfrak{C}_{w} \rightarrow Y$ are closed in $\bar{X}_{\text {aff }}$. The fiber over $y \in Y$ is an orbit of the stabilizer $\operatorname{Stab}_{G \times G}(y)$. Without loss of generality we can assume $y \in Y$ is the image of $e \in G \times G$ and therefore $\operatorname{Stab}_{G \times G}(y)=T_{\Delta} \cdot\left(U_{B} \times U_{B}\right) \subset B \times B$. It is easy to see from the definition of $\mathfrak{C}_{w}$ that the diagonal Cartan subgroup $T_{\Delta}$ stabilizes the point $\tilde{y}$ in the preimage of $y$ in $\mathfrak{C}_{w}$, where $\tilde{y}$ is the image of $e$. Thus this fiber is an orbit of the unipotent group $U_{B} \times U_{B}$. So the lemma follows from Kostant-Rosenlicht Theorem saying that an orbit of the action of a unipotent algebraic group on an affine variety is closed (see e.g. [19, Proposition 2.4.14]).

2.3. Partial compactifications of $Y$. Variety $Y$ is a principal bundle over $\mathcal{B}^{2}$ with structure group $(\mathbb{T} \times \mathbb{T}) / \mathbb{T}_{\Delta} \cong \mathbb{T}$ where $\mathbb{T}_{\Delta} \subset \mathbb{T} \times \mathbb{T}$ is the diagonal subgroup and the last isomorphism comes from the embedding of the first factor to $\mathbb{T} \times \mathbb{T}$.

Recall that $X^{*}(\mathbb{T})^{+}$is the subset of dominant weights in the weight lattice $X^{*}(\mathbb{T})$. For $w \in W$ set $X^{*}(\mathbb{T})_{w}^{+}=w\left(X^{*}(\mathbb{T})^{+}\right)$. We define a partial compactification $\overline{\mathbb{T}}_{w}$ of $\mathbb{T}$ by $\overline{\mathbb{T}}_{w}:=\operatorname{Spec}\left(F\left[X^{*}(\mathbb{T})_{w}^{+}\right]\right) \supset \operatorname{Spec}\left(F\left[X^{*}(\mathbb{T})\right]\right)=\mathbb{T}$. We set $\bar{Y}_{w}=Y \times^{\mathbb{T}} \overline{\mathbb{T}}_{w}=$ $\left(Y \times \overline{\mathbb{T}}_{w}\right) / \mathbb{T}$, where $\mathbb{T}$ acts diagonally; this is a partial compactification of $Y$.

For example, if $G=S L(2)$, then $\bar{Y}_{e}$ is the total space of line bundle $\mathcal{O}(-1,1)$ on $\mathbb{P}^{1} \times \mathbb{P}^{1}$, while $\bar{Y}_{s}$ is the total space of $\mathcal{O}(1,-1)$; here $e, s$ are as in Example 2.4.

2.3.1. Properness of the closure. Let $\overline{\mathfrak{C}}_{w}$ be the closure of $\mathfrak{C}_{w} \subset X \times Y$ in $\bar{X}_{\text {aff }} \times \bar{Y}_{w}$ and $\overline{\mathfrak{C}}_{w}^{\prime}$ the closure of the image of $\mathfrak{C}_{w}$ in $\bar{X}_{\text {aff }} \times \mathcal{B}^{2}$ under the projection

$$
I d_{\bar{X}_{\text {aff }}} \times p_{1,2}^{Y}: \bar{X}_{\text {aff }} \times Y \rightarrow \bar{X}_{\text {aff }} \times \mathcal{B}^{2}
$$

Proposition 2.6. a) The natural map $\overline{\mathfrak{C}}_{w} \rightarrow \overline{\mathfrak{C}}_{w}^{\prime}$ is an isomorphism.

b) The projection $\overline{\mathfrak{C}}_{w} \rightarrow \bar{X}_{\text {aff }}$ is proper.

Proof. Since $\mathcal{B}^{2}$ is proper, b) follows from a).

We start the proof of a) with the following:

Lemma 2.7. a) The space $\bar{Y}_{w}$ is a closed subscheme in the fiber product of total spaces of line bundles on $\mathcal{B}^{2}$ of the form $\mathcal{O}(\lambda,-\lambda), \lambda \in \Lambda_{w}^{+}$.

b) The subscheme $\mathfrak{C}_{w} \subset X \times Y$ is the graph of sections of the pull-back of those line bundles to a subvariety $Z \subset X \times \mathcal{B}^{2}$; here $Z$ is the preimage under the projection $X \times \mathcal{B}^{2} \rightarrow \mathcal{B}^{4}$ of the subvariety $\left\{\left(B_{1}, B_{2}, B_{3}, B_{4}\right) \mid B_{1} \stackrel{w}{-} B_{3}, B_{2} \stackrel{w_{0} w}{-} B_{4}\right\}$.

Proof. a) is clear since $\overline{\mathbb{T}}_{w}$ is a closed subscheme in a product of affine lines, where the torus $\overline{\mathbb{T}}$ acts on each of the line by a character in $X^{*}(T)_{w}^{+}$. Statement (b) amounts to the map $X \times Y \rightarrow X \times \mathcal{B}^{2}$ inducing an isomorphism $\mathfrak{C}_{w} \rightarrow Z$. It maps $\mathfrak{C}_{w}$ to $Z$ by Lemma[2.3(a); now the map $\mathfrak{C}_{w} \rightarrow Z$ is an isomorphism since the action of $G \times G$ on both $\mathfrak{C}_{w}$ and $Z$ is easily seen to be simply transitive.

Now Proposition 2.6(a) would follow if we show that the sections described in Lemma 2.7(b) extend to the closure of $Z$ in $\bar{X}_{\text {aff }} \times \mathcal{B}^{2}$. Since $\bar{X}_{\text {aff }}$ is the affine closure of a smooth quasi-affine variety $X$, it is normal and $\bar{X}_{\text {aff }} \backslash X \subset \bar{X}_{\text {aff }}$ is of 
codimension at least two, so any section of the line bundle on $X \times \mathcal{B}^{2}$ extends to $\bar{X}_{\text {aff }} \times \mathcal{B}^{2}$. Thus it suffices to show that the sections of our line bundles extend to the closure of $Z$ in $X \times \mathcal{B}^{2}$.

Since the sections are $G \times G$ invariant and $X$ is a homogeneous space, the desired statement follows from the next lemma.

Lemma 2.8. Fix $w \in W$. For a weight $\lambda \in X^{*}(\mathbb{T})$ the following are equivalent:

i) $\lambda \in X^{*}(\mathbb{T})_{w}^{+}$.

ii) The line bundle $\left.\mathcal{O}(\lambda,-\lambda)\right|_{\overline{\mathcal{B}_{w_{0} w}} \times \overline{\mathcal{B}_{w}}}$ has a nonzero $U \times U$ invariant section.

Proof. Let us recall a description of the divisor of a $U$-invariant section of $\mathcal{O}(\lambda)$ on $\mathcal{B}_{w}$ viewed as a rational section on $\overline{\mathcal{B}_{w}}$; see e.g. [4, Proposition 1.4.5] and references therein. Namely, for $w \in W$ components of codimension 1 in $\overline{\mathcal{B}_{w}} \backslash \mathcal{B}_{w}$ are in bijection with reflections $s_{\alpha}$, where $\alpha$ is a positive not necessarily simple coroot, such that $\ell\left(w s_{\alpha}\right)=\ell(w)-1$. The multiplicity of the corresponding component in the divisor of a $U$-invariant section of $\left.\mathcal{O}(\lambda)\right|_{\overline{\mathcal{B}_{w}}}$ is then equal to $\langle\lambda, \alpha\rangle$. Thus those $\alpha$ for which $\ell\left(w s_{\alpha}\right)=\ell(w)+1$ correspond to elements of $w^{-1}(\Phi)$ which are positive roots; while those $\alpha$ for which $\ell\left(w_{0} w s_{\alpha}\right)=\ell\left(w_{0} w\right)+1$ correspond to elements of $w^{-1}(\Phi)$ which are negative roots. We see that a nonzero $U$ invariant section exists both for $\mathcal{O}(\lambda)$ on $\overline{\mathcal{B}_{w}}$ and for $\mathcal{O}(-\lambda)$ on $\overline{\mathcal{B}_{w_{0} w}}$ if and only if $\lambda$ is positive on $w^{-1}(\Phi)$, i.e., $\lambda \in X^{*}(\mathbb{T})_{w}^{+}$.

2.4. The wonderful compactification. We introduce a version of De ConciniProcesi's wonderful compactification relevant for our purposes. If $G$ is of adjoint type, we let $\bar{G}$ be the wonderful compactification [9] (see also [11, [18] and [6, §6.1]). If $G$ is an arbitrary reductive group we let $G_{a d}$ denote the quotient of $G$ by its center, and let $G^{\prime} \subset G$ be the commutator subgroup, and consider the homomorphism $G \rightarrow\left(G / G^{\prime}\right) \times G_{a d}$ which is surjective and has a finite kernel. We let $\bar{G}$ denote normalization of $\left(G / G^{\prime}\right) \times \overline{G_{a d}}$ in $G$.

Notice that by [6. Proposition 6.2.4] a normal equivariant partial compactification of $G$ with an equivariant morphism to $\bar{G}_{a d}$ (also known as a toroidal $G$ embedding) is uniquely determined by the toric variety which is the closure $\bar{T}$ of a maximal torus $T$ of $G$ in $\bar{G}$. It is easy to see (cf. [6, Lemma 6.1.6]) that in our case $\bar{T}$ is the toric variety corresponding to the fan coming from the Weyl chambers stratification.

The components of $\partial G:=\bar{G} \backslash G$ are indexed by $\Sigma$, this easily follows e.g. from [6. Proposition 6.2.3(ii)]. For $i \in \Sigma$ let $\bar{G}_{i}$ be the corresponding component.

If $G$ is a product of a torus and an adjoint group, then $\bar{G}$ is smooth and $\partial G=$ $\bar{G} \backslash G$ is a divisor with normal crossings (see e.g. [6, Theorem 6.1.8]); this is not necessarily true in general.

For $I \subset \Sigma$ set $\bar{G}_{I}=\bigcap_{i \in I} \bar{G}_{i}$. Let $G_{I}$ be the complement in $\bar{G}_{I}$ to the union of $\bar{G}_{J}, J \supsetneq I$. By convention $\bar{G}_{\emptyset}=\bar{G}$ and $G_{\emptyset}=G$.

Claim 2.9. a) The action of $G \times G$ on $G_{I}$ is transitive.

b) Let $P_{I}=L_{I} U_{I}$ be a parabolic in the conjugacy class corresponding to $I$, let $P^{-}=L_{I} U_{I}^{-}$be an opposite parabolic. Then there exists a point in $G_{I}$ with stabilizer $H_{I}:=\left\{\left(u l, u^{-} l^{-}\right) \mid u \in U_{I}, u^{-} \in U_{I}^{-}, l, l^{-} \in L_{I}, l^{-1} l^{-} \in Z\left(L_{I}\right)^{0}\right\}$, where $Z\left(L_{I}\right)^{0}$ is the identity component in the center $Z\left(L_{I}\right)$ of $L_{I}$.

Proof. a) appears, for example, as [6, Proposition 6.2.3(iii)]. Statement b) for $G$ adjoint is [18, Lemma 1(ii)], notice that in this case $Z\left(L_{I}\right)^{0}=Z\left(L_{I}\right)$. The general 
case follows: using the adjoint case we find a point $x_{I} \in \bar{G}$ whose stabilizer is a finite index subgroup in $\left\{\left(u l, u^{-} l^{-}\right) \mid u \in U_{I}, u^{-} \in U_{I}^{-}, l, l^{-} \in L_{I}, l^{-1} l^{-} \in Z\left(L_{I}\right)\right\}$; in view of [6. Lemma 6.1.4(i)] we can assume without loss of generality that $x_{I}$ lies in the closure $\bar{T}$ of a maximal torus $T \subset L_{I}$, then the above description of $\bar{T}$ shows that the stabilizer of $x_{I}$ in $T$ is connected, thus that the stabilizer of $x_{I}$ in $G \times G$ is connected and coincides with $H_{I}$.

See also [11, Theorem 2.22, Proposition 2.25] for the case of an adjoint group over a field of characteristic zero.

2.4.1. Normal bundles to strata. The isomorphism $G_{I}=(G \times G) / H_{I}$ shows that we have a canonical map $X_{I} \rightarrow G_{I}$ which is a principal bundle with the structure group $Z\left(L_{I}\right)^{0}$.

For a locally closed smooth subvariety $Z$ in a smooth variety $M$ let $N_{M}(Z)$ denote the normal bundle. More generally, in the situation described in the Appendix, section 11.1, we let $N_{M}(Z)$ denote the quasi-normal cone in the sense of 11.1 .

If $G$ is adjoint, the normal bundle $N_{\bar{G}}\left(\bar{G}_{I}\right)$ splits canonically as a sum of line bundles $\left.N_{\bar{G}}\left(\bar{G}_{i}\right)\right|_{\bar{G}_{I}}, i \in I$. We have an action of $Z\left(L_{I}\right)$ on $N_{\bar{G}}\left(\bar{G}_{I}\right)$ such that the action on $\left.N_{\bar{G}}\left(\bar{G}_{i}\right)\right|_{\bar{G}_{I}}, i \in I$ is by the character $\alpha_{i}$. We will need a generalization of this observation to an arbitrary $G$.

Let $A_{I}$ denote the closure of $Z\left(L_{I}\right)^{0}$ in the $\overline{\mathbb{T}}_{\text {aff }}:=\operatorname{Spec}\left(F\left[\Lambda^{+}\right]\right)$. This is a toric variety for the torus $Z\left(L_{I}\right)^{0}$.

Claim 2.10. a) The fibration $N_{\bar{G}}\left(G_{I}\right) \rightarrow G_{I}$ is canonically isomorphic to the bundle with fiber $A_{I}$ associated to the principal $Z\left(L_{I}\right)^{0}$ bundle $X_{I} \rightarrow G_{I}$.

b) There exists a canonical $G \times G \times Z\left(L_{I}\right)$ equivariant open embedding $X_{I} \rightarrow$ $N_{\bar{G}}\left(G_{I}\right)$. Its image is the complement to the divisor $\bigcup_{i \in I} N_{\bar{G}_{i}}\left(G_{I}\right)$.

Proof. (b) follows from (a), while (a) follows from Theorem 11.3(c) in view of Example 11.2. Alternatively, part (a) can be deduced from [6. Proposition 6.2.3(i)] which shows that the variety $\bar{G}$ is locally isomorphic to the product of the affine toric variety $\overline{\mathbb{T}}_{\text {aff }}$ with a smooth variety, the stratification of $\bar{G}$ by $G \times G$ orbits $G_{I}$ corresponds to the stratification by $\mathbb{T}$ orbits on $\overline{\mathbb{T}}_{\text {aff }}$.

Remark 2.11. The following description of $X_{I}$, though not used explicitly in this paper, is closely related to Claim 2.10. The space $X_{I}$ is quasi-affine, it is the dense $G \times G$ orbit in its affine closure $\bar{X}_{I, \text { aff }}$. Thus $X_{I}$ can be reconstructed from the algebra of global regular functions $\mathcal{O}_{g l}\left(X_{I}\right)$. Assume for simplicity that $F$ is of characteristic zero. Then the space $\mathcal{O}_{g l}(G)$ is isomorphic as a $G \times G$-module to: $\bigoplus_{\lambda \in X^{*}(\mathbb{T})^{+}} E_{\lambda}$, where $E_{\lambda}=V_{\lambda} \otimes V_{\lambda}^{*}$ for the representation $V_{\lambda}$ with highest weight $\lambda$. Let $m_{\lambda, \mu}^{\nu}: E_{\lambda} \otimes E_{\mu} \rightarrow E_{\nu}$ be the corresponding component of multiplication in $\mathcal{O}_{g l}(G)$. Then $\mathcal{O}_{g l}\left(X_{I}\right)=\mathcal{O}_{g l}(G)$ as $G \times G$ modules and multiplication map in $\mathcal{O}_{g l}\left(X_{I}\right)$ is the sum of maps $m_{\lambda, \mu}^{\nu}(I): E_{\lambda} \otimes E_{\mu} \rightarrow E_{\nu}$, where $m_{\lambda, \mu}^{\nu}(I)=m_{\lambda, \mu}^{\nu}$ when $\lambda+\mu-\nu$ is trivial on $Z\left(L_{I}\right)$ and $m_{\lambda, \mu}^{\nu}(I)=0$ otherwise.

Remark 2.12. The correspondence $\mathfrak{C}_{w}$ can also be described in terms of geometry of the wonderful compactification $\bar{G}$. Namely, let $\Gamma \subset G \times \mathcal{B}^{2}$ be the graph of the action of $G$ on $\mathcal{B}$, i.e., it is given by $\Gamma=\left\{\left(g, B_{1}, B_{2}\right) g\left(B_{1}\right)=B_{2}\right\}$. Let $\bar{\Gamma}$ be the closure of $\Gamma$ in $\bar{G} \times \mathcal{B}^{2}$. Let $Z \cong \mathcal{B}^{2} \subset \bar{G}$ be the closed $G^{2}$ orbit. According to [5], [7], the subset

$$
\left(Z \times \mathcal{B}^{2}\right)_{w}:=\left\{\left(B_{1}, B_{2} ; B_{3}, B_{4}\right) \mid B_{1} \stackrel{w}{-} B_{3}, B_{2} \stackrel{w_{0} w}{-} B_{4}\right\}
$$


is an open smooth subscheme in $\bar{\Gamma} \cap Z \times \mathcal{B}^{2}$. We claim that $\mathfrak{C}_{w}$ is canonically isomorphic to an open part in the quasi-normal cone (see Appendix for a definition of this term) $N_{\bar{\Gamma}}\left(\left(Z \times \mathcal{B}^{2}\right)_{w}\right)$. The projection $\mathfrak{C}_{w} \rightarrow X$ is the restriction of the natural map (the normal differential of the projection, i.e., the composition of the differential with projection to the normal bundle) $N_{\bar{\Gamma}}\left(\left(Z \times \mathcal{B}^{2}\right)_{w}\right) \rightarrow N_{\bar{G}}(Z) \supset X$. The projection $\mathfrak{C}_{w} \rightarrow Y$ can be described as follows. Recall that there exists a canonical action map $a: \Gamma=G \times \mathcal{B} \rightarrow Y, a:(g, B) \rightarrow(\tilde{B}, g(\tilde{B}) \bmod T)$, where $\tilde{B}$ is an arbitrary lifting of $B \in \mathcal{B}$ to a point in $G / U$. Let us view $a$ as a rational map $\bar{\Gamma} \rightarrow \bar{Y}_{w}$. We claim that $\left(Z \times \mathcal{B}^{2}\right)_{w}$ is contained in the domain of definition of this map, and $a:\left(Z \times \mathcal{B}^{2}\right)_{w} \rightarrow \mathcal{B}^{2} \subset \bar{Y}_{w}$. Thus the normal differential of $a$ gives a map $N_{\bar{\Gamma}}\left(\left(Z \times \mathcal{B}^{2}\right)_{w}\right) \rightarrow N_{\mathcal{B}^{2}}\left(\bar{Y}_{w}\right)=\bar{Y}_{w}$, which restricts to the projection $\mathfrak{C}_{w} \rightarrow Y$.

We neither prove nor use these statements.

2.4.2. Quotient by the $U_{P}$ action. Let $P=P_{I}=L_{I} U_{I}$ be a parabolic subgroup whose conjugacy class corresponds to $I \subset \Sigma$, let $Z_{I}=Z\left(L_{I}\right)^{0}$ denote the neutral component in the center of $L_{I}$.

The map $G / U_{I} \rightarrow G /\left(U_{I} Z\left(L_{I}\right)^{0}\right)$ is a principal $Z\left(L_{I}\right)^{0}$ bundle. We let $\overline{G / U_{I}}:=$ $\left(A_{I} \times G / U_{I}\right) / Z_{I}$ be the associated bundle over $G /\left(U_{I} Z_{I}\right)$ with fiber $A_{I}$.

In view of Claim 2.9 (b) we have a canonical projection $G_{I} \rightarrow G / P_{I}^{-}$; let $G_{I}^{0} \subset G_{I}$ be the preimage of the open $U_{I}$ orbit under that projection.

Consider the closure of the subgroup $Z_{I}$ in $\bar{G}$. This is a toric variety for the torus $Z_{I}$.

Claim 2.13. a) The closure of $Z_{I}$ intersects $G_{I}^{0}$ at a unique point, which we denote by $z$.

b) Let $\tilde{Z}_{I}$ be the open subvariety in this toric variety which is the union of all $Z_{I}$-orbits whose closure contains $z$. Let $\bar{G}^{0}(I)$ denote the image of $\tilde{Z}_{I}$ under the action of $G \times U_{I}$. Then the subset $\bar{G}^{0}(I) \subset \bar{G}$ is an open subvariety, such that:

i) $\bar{G}^{0}(I)$ is invariant under the left action of $G$ and the right action of $U_{I}$, the action of $U_{I}$ on $\bar{G}^{0}(I)$ is free.

ii) The quotient $\bar{G}^{0}(I) / U_{I}$ is isomorphic to $\overline{G / U_{I}}$.

iii) We have $G \subset \bar{G}^{0}(I), G_{I}^{0} \subset \bar{G}^{0}(I)$ and the induced embeddings of the quotient spaces coincide respectively with the tautological embedding $G / U_{I} \rightarrow \overline{G / U_{I}}$ and the embedding $G /\left(U_{I} Z_{I}\right) \rightarrow \overline{G / U_{I}}$ induced by the embedding $\{0\} \rightarrow A_{I}$.

Proof. Fix a maximal torus $T \supset Z_{I}$ and Borel subgroups $B=T U \subset P_{I}$ and $B^{-}=T U^{-} \subset P_{I}^{-}$. According to [6, Proposition 6.2.3(i)] there exists an open subvariety $\mathfrak{U} \subset \bar{G}$ (denoted by $X_{0}$ in loc. cit.) such that the action of $U^{-} \times U$ on $\mathfrak{U}$ is free, where $U^{-}$acts on the left and $U$ acts on the right. Furthermore, $\mathfrak{U} \cong U^{-} \times \overline{\mathbb{T}}_{\text {aff }} \times U$ (where $\overline{\mathbb{T}}_{\text {aff }}=A_{\Sigma}$ is as in the proof of Claim 2.10, in loc. cit. it is denoted by $X_{0}^{\prime}$ ). By [6, Proposition 6.2.3(ii)] the intersection $\overline{\mathbb{T}}_{\text {aff }} \cap \bar{G}_{I}$ is a $T$ orbit, to be denoted by $\overline{\mathbb{T}}_{\text {aff }}(I)$. The closure of $Z_{I}$ in $\overline{\mathbb{T}}_{\text {aff }}$ (recall that it is denoted by $\left.A_{I}\right)$ intersects the $T$-orbit $\overline{\mathbb{T}}_{\text {aff }}(J)$ iff $J \supseteq I$. Also the intersection of this closure with $\overline{\mathbb{T}}_{\text {aff }}(I)$ consists of a single point $z$. This point $z \in G_{I}$ lies on a free $U^{-} \times U$ orbit, hence it lies in $\bar{G}^{0}(I)$. Conversely, any other point $z^{\prime}$ in the intersection of $G_{I}$ with the closure of $Z_{I}$ is stabilized by the diagonal action of $L_{I}$, as well as by a subgroup which is conjugate to but different from $U_{I} \times U_{I}^{-}$and is normalized by 
$L_{I}$, hence $z^{\prime}$ does not lie in $G_{I}^{0}$. This proves (a). Notice also that we have shown that $A_{I} \cong \tilde{Z}_{I}$.

To check that $\bar{G}_{0}(I)$ is open it is enough to show that it contains a neighborhood of $Z_{I}$. For such a neighborhood we can take the image of $\tilde{Z}_{I}$ under the action of $U^{-} \times T \times U$ : on the one hand it coincides with the $\bigcup_{J \supseteq I} U^{-} \overline{\mathbb{T}}_{\text {aff }}(J) U$ which is open in $\mathfrak{U}$, hence in $\bar{G}$; on the other hand, it is contained in $\bar{G}^{0}(I)$ since $B \subset L_{I} \cdot U_{I}$ and $L_{I}$ commutes with $Z_{I}$.

Since $\tilde{Z}_{I}$ is contained in $\mathfrak{U}$, it lies in the union of free (right) $U_{I}$ orbits, so the right action of $U_{I}$ on $\bar{G}_{0}(I)$ is free. The image of the map $A_{I} \cong \tilde{Z}_{I} \mapsto \bar{G}_{0}(I) / U_{I}$ is clearly invariant under $U_{I} Z_{I}$ and the action of $U_{I}$ on this image is trivial, while the action of $Z_{I}$ is the canonical one. Thus we get a surjective map $\overline{G / U_{I}} \rightarrow \bar{G}^{0}(I) / U_{I}$. The stabilizer of any point in $\tilde{Z}_{I}$ under the left action of $G$ is contained in $U_{I}$, hence the stabilizer of any point in the image of $\tilde{Z}_{I}$ under the map to $\bar{G}^{0}(I) / U_{I}$ equals $U_{I}$, this implies that the map $\overline{G / U_{I}} \rightarrow \bar{G}^{0}(I) / U_{I}$ is an isomorphism. Property (iii) is clear from the construction.

Example 2.14. Let $G=P G L(2)$, thus $\bar{G}=\mathbb{P}(\operatorname{End}(V))$ where $V=k^{2}$ is the twodimensional vector space. Let $P_{I}=B_{I}$ be the group of upper triangular matrices. Then $\bar{G}^{0}(I)$ is the projectivization of the set of matrices with a nonzero second column. The quotient $\bar{G}^{0}(I) / U_{I}$ maps to $\mathbb{P}^{1}$ by the map sending a matrix to the line of its second column, this identifies $\bar{G}^{0}(I) / U_{I}$ with the total space of the line bundle $\mathcal{O}_{\mathbb{P}^{1}}(2)$.

2.4.3. Compactified Bruhat cells. Fix $B \in \mathcal{B}$. For $w \in W$ let $G_{w} \subset G$ denote the corresponding $B \times B$ orbit. Let $\overline{G_{w}}$ denote the closure of $G_{w}$ in $\bar{G}$.

To unburden notations we state the next claim for a semi-simple group $G$, the answer in the general case differs by replacing $\mathcal{B}^{2}$ and its subvarieties by their product with the torus $G / G^{\prime}$.

Claim 2.15. a) The components of the intersection of $\overline{G_{w}}$ with the closed stratum $\mathcal{B}^{2} \subset \bar{G}$ are in bijection with pairs $w_{1}, w_{2}$ such that $\ell(w)+\ell\left(w_{0}\right)=\ell\left(w_{1}\right)+\ell\left(w_{2}\right)$.

b) Given such a pair $\left(w_{1}, w_{2}\right)$, the smooth part of the corresponding component contains the $B \times B$ orbits $\mathcal{B}_{w_{1}} \times \mathcal{B}_{w_{2}}$.

c) The open part of the quasi-normal cone to the smooth locus of the corresponding component is identified with $X_{w_{1}, w_{2}}$ where $X_{w_{1}, w_{2}}$ is the corresponding $B \times B$ orbit in $X$.

Proof. If $G$ is adjoint, then (a), (b) follow from [5, Theorem 2.1(ii)]. In view of Claim 2.10, part (c) in this case follows from [5, Theorem 2.1(i)] which implies that the normal bundle to $\overline{G_{w}} \cap \mathcal{B}^{2}$ in $\overline{G_{w}}$ is isomorphic to the restriction of the normal bundle to $\mathcal{B}^{2}$ in $\bar{G}$. The general case follows since $\overline{G_{w}}$ is easily shown to coincide with the preimage of of $\overline{\left(G_{a d}\right)_{w}}$, this implies in particular that the quasi-normal cone to the smooth part of $\overline{G_{w}} \cap \mathcal{B}^{2}$ is the fiber product of the quasi-normal cone to $\mathcal{B}^{2}$ in $\bar{G}$ by the smooth part of $\overline{G_{w}} \cap \mathcal{B}^{2}$.

Corollary 2.16. For $w, w_{1}, w_{2}$ as in Claim 2.15 we have a canonical isomorphism of $\mathbb{T}$ torsors: $U_{B} \backslash G_{w} / U_{B} \cong U_{B} \backslash X_{w_{1}, w_{2}} / B_{U}$.

Proof. It easy to see that for a smooth toric variety $C$ the normal bundle to a torus orbit is canonically identified with an open subvariety in $C$. The same is true for 
a not necessarily smooth normal toric variety with a fixed finite equivariant map to a smooth one, where instead of the normal bundle we consider the quasi-normal cone introduced in the Appendix.

One can apply this to the closure $C_{w}$ of a generic orbit of a maximal torus $T$ acting on $G_{w}$ by left translations and the $T$ orbit $C_{w_{1}, w_{2}}:=C \cap \mathcal{B}_{w_{1}} \times \mathcal{B}_{w_{2}}$. The open orbit of $T$ on $C_{w}$ maps isomorphically to $U_{B} \backslash G_{w} / U_{B}$, while the open orbit in $N_{C_{w_{1}, w_{2}}}(C)$ maps isomorphically to $U_{B} \backslash X_{w_{1}, w_{2}} / U_{B}$. Thus the sought for isomorphism is obtained by restricting the isomorphism described in the previous paragraph to the open $T$ orbit.

\subsection{More on partial compactifications of $X$.}

2.5.1. Torus closure. Let $\overline{\mathbb{T}}:=\overline{\mathbb{T}}_{1}$ denote the partial compactification of the abstract Cartan $\mathbb{T}$ attached to the unit element in $W$ by the construction of section 2.3 .

Claim 2.17. a) The closure in $\bar{X}_{\text {aff }}$ of any orbit of the abstract Cartan $\mathbb{T}$ acting on $X$ is isomorphic to $\overline{\mathbb{T}}$.

b) For any $w_{1}, w_{2}$ there exists a $\mathbb{T}$ equivariant isomorphism $\mathbb{T} \cong X_{w_{1}, w_{2}} / U_{B}^{2}$. The corresponding projection $X_{w_{1}, w_{2}} \rightarrow \mathbb{T}=X_{w_{1}, w_{2}} / U_{B}^{2}$ extends to a regular map $\overline{X_{w_{1}, w_{2}}} \rightarrow \overline{\mathbb{T}}$ where $\overline{X_{w_{1}, w_{2}}}$ denotes the closure in $\bar{X}_{\text {aff }}$.

Proof. It is clear that the weights of $\mathbb{T}$ acting on the space of regular functions on $X$ are precisely the dominant weights; also, given such a weight $\lambda$ and an orbit of $\mathbb{T}$ there exists a function transforming by the character $\lambda$ with a nonzero restriction to the orbit. This implies part (a). The first statement in part (b) is clear, the second one amounts to existence for every dominant weight $\lambda$ of a section of the line bundle $\mathcal{O}(\lambda, \lambda)$ on $\mathcal{B}^{2}$ whose restriction to $\mathcal{B}_{w_{1}} \times \mathcal{B}_{w_{2}}$ is nonzero and $U_{B}^{2}$ invariant. Since $U_{B}^{2}$ is unipotent it suffices to see existence of a section with a nonzero restriction which is clear.

2.5.2. Embedding into the matrix space and its compactification. For a vector space $\mathcal{V}$ let $\mathbb{P}(\mathcal{V})$ denote the projectivization of $\mathcal{V}$, let $\tilde{\mathbb{P}}(\mathcal{V})=\mathbb{P}(\mathcal{V} \oplus F)=\mathbb{P}(\mathcal{V}) \cup \mathcal{V}$ be its projective compactification, and set $\mathcal{V}^{0}=\mathcal{V} \backslash\{0\}, \tilde{\mathbb{P}}(\mathcal{V})^{0}=\tilde{\mathbb{P}}(\mathcal{V}) \backslash\{0\}$.

We set $\bar{X}=N_{\bar{G}}\left(\bar{G}_{\Sigma}\right)$; in view of Claim 2.10 it is identified with the fibration over $G_{\Sigma}$ associated to the $\mathbb{T}$-bundle $X \rightarrow G_{\Sigma}$ and the $\mathbb{T}$ space $\overline{\mathbb{T}}_{\text {aff }}$. In other words, $\bar{X}$ is the relative spectrum of the sheaf $\bigoplus_{\lambda \in \Lambda^{+}} \mathcal{O}_{\mathcal{B}}(\lambda) \otimes \mathcal{O}_{\mathcal{B}}(\check{\lambda})$ of commutative rings on $\mathcal{B}^{2}$ where $\check{\lambda}=-w_{0}(\lambda)$ is the dual dominant weight.

Claim 2.18. a) Let $M$ be a $G$-module. Then there exists a canonical map $\rho_{M}$ : $X \rightarrow \operatorname{End}(M)$. This map extends to a map $\overline{\rho_{M}}: \bar{X} \rightarrow \tilde{\mathbb{P}}(\operatorname{End}(M))^{0}$.

b) There exists a finite collection of $G$-modules $M_{i}$, such that the map $\prod \overline{\rho_{M_{i}}}$ : $\bar{X} \rightarrow \prod \tilde{\mathbb{P}}\left(\operatorname{End}\left(M_{i}\right)\right)^{0}$ is a closed embedding. This embedding sends the zero section of $N_{\bar{G}}\left(\bar{G}_{\Sigma}\right)=\bar{X}$ to $\prod \mathbb{P}\left(\operatorname{End}\left(M_{i}\right)\right)$.

Proof. Fix a pair of opposite Borels $B=T U, B^{-}=T U^{-}$, so that $X=(G / U \times$ $\left.G / U^{-}\right) / T$. Let $\lambda_{i}$ be the set of highest weights of $M$ (i.e. maximal elements in the set of weights of $M)$ and let $E_{\lambda_{i}} \in \operatorname{End}(M)$ be $T$-invariant projection to the corresponding weight space. The operator $E=\sum_{i} E_{\lambda_{i}}$ is invariant under the left action of $U$, the right action of $U^{-}$and the diagonal action of $T$, thus we get a map $\rho_{M}: X \rightarrow \operatorname{End}(M)$ sending the unit coset to $E$. Since any two pairs of opposite Borel subgroups are conjugate by an element of $G$ which is defined uniquely up to 
multiplication by an element in the Cartan subgroup, the map $X \rightarrow \operatorname{End}(M)$ does not depend on the auxiliary choice. The extension to map $\overline{\rho_{M}}: \bar{X} \rightarrow \tilde{\mathbb{P}}(\operatorname{End}(M))$ comes from the standard highest weight morphism of $G^{2}$ equivariant bundles over $\mathcal{B}^{2}: M^{*} \otimes M \otimes \mathcal{O}_{\mathcal{B} \times \mathcal{B}} \rightarrow \bigoplus_{i} \mathcal{O}\left(\lambda_{i}, \check{\lambda}_{i}\right)$; this proves part (a). According to 6, Lemma 6.1.1] there exists a module $M_{0}$ for which the map $\mathcal{B} \times \mathcal{B} \rightarrow \mathbb{P}\left(\operatorname{End}\left(M_{0}\right)\right)$ induced by $\rho_{M_{0}}$ is a closed embedding. We can choose modules $M_{1}, \ldots, M_{k}$ and a highest weight $\lambda_{i}$ of $M_{i}$ so that the weights $\lambda_{1}, \ldots, \lambda_{k}$ generate the lattice of dominant weights. Then the map $\prod_{i=0}^{k} \overline{\rho_{M_{i}}}$ is easily seen to be a closed embedding. The last property stated in (b) is clear from the construction.

\section{CO-SPECIALIZATON FROM NORMAL BUNDLE FOR FUNCTIONS ON A $p$-ADIC MANIFOLD}

From now on $F$ is a non-Archimedian local field with the ring of integers $O \subset F$ and a uniformizer $\pi$. From now on topological notions are in reference to the $F$-topology on the space of $F$-points of an algebraic variety over $F$ or its subspaces.

For a totally disconnected space $X$ we let $\mathcal{S}(X)$ denote the space of locally constant compactly supported functions on $X$.

Let $\mathrm{W}$ be a smooth analytic variety over a non-Archimedian local field $F, D \subset$ $\mathrm{W}$ an open subset such that the complement $\mathrm{W} \backslash D$ is a union $S=\bigcup_{i \in \Sigma} S_{i}$ of smooth divisors $S_{i}$ with normal crossing. For $I \subset \Sigma$ we define $S_{I}=\bigcap_{i \in I} S_{i}$, $S_{I}^{0}:=S_{I}-\bigcup_{j \in \Sigma-I} S_{I \cup j}$ and denote by $r_{I}: N_{I} \rightarrow S_{I}$ the normal bundle to $S_{I}$. For any $J \subset I$ we denote by $N_{I}^{J} \subset N_{I}$ the normal bundle to $S_{I}$ in $S_{J}$. Locally in $S_{I}$ we can identify $N_{I}$ with the product $\mathbb{A}^{I} \times S_{I}$.

The following is immediate.

Claim 3.1. a) We have a canonical direct sum decomposition

$$
N_{I}=\bigoplus_{i \in I} N_{I}^{I-\{i\}}
$$

b) $r_{J}^{*}\left(N_{I}^{J}\right)$ is the normal bundle to $r_{J}^{-1}\left(S_{I}\right)$ in $N_{J}$.

c) The complement $N_{I}-r_{I}^{-1}\left(S_{I}^{0}\right)$ is a union of smooth divisors $r_{I}^{-1}\left(S^{I \cup j}\right), j \in$ $\Sigma \backslash I$ with normal crossing.

Definition 3.2. Let $U \subset \mathrm{W}$ be an open subset. We say that an analytic open embedding $\tau_{I}: U \rightarrow N_{I}$ is admissible if:

$\tau_{I \mid S_{I} \cap U}=I d$,

$\tau_{I}\left(U \cap S_{J}\right) \subset N_{I}^{J}$ for $J \subset I$ and

the normal component of $d \tau_{I \mid S_{I} \cap U}$ equals the canonical projection $\left.T \mathrm{~W}\right|_{S_{I}} \rightarrow N_{I}$.

Any admissible map $\tau_{I}: U \rightarrow N_{I}$ defines an embedding $\tau_{I V}^{*}: \mathcal{S}(V) \rightarrow \mathcal{S}(U)$ for any open subset $V$ of $\tau_{I}(U) \subset N_{I}$.

We denote by $(a, y) \rightarrow$ ay the natural action of the group $\mathbb{G}_{m}^{I}$ on the bundle $\underline{p}_{I}: N_{I} \rightarrow S_{I}$ and define $X_{I} \subset N_{I}$ as the open subvariety of points with trivial stabilizer; thus $X_{I}=N_{I} \backslash \bigcup_{j \in I} N_{I}^{\{j\}}$.

Definition 3.3. a) For any $\lambda \in \operatorname{Hom}\left(\mathbb{G}_{m}, \mathbb{G}_{m}^{I}\right)=\mathbb{Z}^{I}$ we write $z_{\lambda}:=\lambda\left(\pi^{-1}\right) \in$ $\left(F^{\times}\right)^{I}, z_{I}:=z_{(1, \ldots, 1)}$. 
b) For any $\lambda \in \mathbb{Z}^{I}$ we define an operator $T^{\lambda} \in \operatorname{End}\left(\mathcal{S}\left(N_{I}\right)\right)$ by

$$
T^{\lambda}(f)(y):=f\left(z_{\lambda} y\right) .
$$

For $\lambda=\left(\lambda_{i}\right), \mu=\left(\mu_{i}\right) \in \mathbb{Z}^{I}$ we will say that $\lambda \geq \mu$ if $\lambda_{i} \geq \mu_{i}$ for all $i \in I$.

Lemma 3.4. For any $f \in \mathcal{S}\left(N_{I}\right)$ and a pair $\tau_{I 1}, \tau_{I 2}: U \rightarrow N_{I}$ of admissible maps there exists $\lambda_{0} \in \mathbb{Z}^{I}$ such that $\operatorname{supp}\left(T^{\mu}(f)\right) \subset \tau_{I 1}(U) \cap \tau_{I 2}(U)$ and $\tau_{I}^{\star}\left(T^{\mu}(f)\right)=$ $\tau_{I}^{\star}\left(T^{\mu}(f)\right)$ for all $\mu \geq \lambda_{0}$.

Proof. The following obvious claim implies the lemma.

Let $S$ be an analytic $F$-variety, $\tilde{S}:=F^{n} \times S$ and let $\tau: \tilde{S} \rightarrow \tilde{S}$ be an analytic morphism such that:

a) $\left.\tau\right|_{\{0\} \times S}=I d$.

b) $\left.d \tau\right|_{\{0\} \times s}$ induces the identity map $F^{n} \rightarrow F^{n}$ for every $s \in S$.

Let $t \in\left(F^{\times}\right)^{n}$ be such that $\left|t_{i}\right|<1$. Consider the sequence of morphsims

$$
\phi_{n}: \tilde{S} \rightarrow \tilde{S}, \phi_{n}(\tilde{s}):=t^{-n} \tau_{I}\left(t^{n} \tilde{s}\right) \text {, where } t(v, s):=(t v, s) .
$$

Claim 3.5. We have $\phi_{n}(x) \rightarrow x$ as $n \rightarrow \infty$ for every $x \in \tilde{S}$. Moreover, $\phi_{n} \rightarrow I d$ uniformly on every compact subset in $\tilde{S}$.

Definition 3.6. For a set $\mathfrak{S}$ consider the set of partially defined maps $\mathbb{Z}^{I} \rightarrow \mathfrak{S}$ whose domain contains a set of the form $\left\{\lambda \mid \lambda \geq \lambda_{0}\right\}$ for some $\lambda_{0} \in \mathbb{Z}^{I}$. We say that two such maps agree asymptotically if they coincide on $\left\{\lambda \mid \lambda \geq \lambda_{0}\right\}$ for $\lambda_{0} \in \mathbb{Z}^{I}$. This defines an equivalence relation on the set of such maps, let $\mathfrak{S}_{\text {as }}(I)$ denote the set of equivalence classes.

We restate the result of Lemma 3.4 by saying that we have a well-defined asymptotic embedding of

$$
\mathcal{S}\left(N_{I}\right) \rightarrow \mathcal{S}(\mathrm{W})_{\text {as }}(I), \quad f \mapsto\left(\lambda \mapsto \tau_{I}^{\star}\left(T^{\lambda}(f)\right)\right) .
$$

Given two subsets $J \subset I \subset \Sigma$ we can apply Lemma 3.4 to the case considered in Claim 3.1 to obtain the asymptotic embedding

$$
j_{I}^{J}: \mathcal{S}\left(N_{I}\right) \rightarrow \mathcal{S}\left(N_{J}\right)_{\text {as }}(I \backslash J), \quad f \mapsto\left(\lambda \mapsto \tau_{I}^{\star}\left(T^{\tilde{\lambda}}(f)\right) .\right.
$$

The following is immediate.

Claim 3.7. For any $f \in \mathcal{S}\left(N_{I}\right)$ we have

$$
j_{J}\left(j_{I}^{J}(f)(\tilde{\lambda})\right)(\mu)=j_{I}(f)(\tilde{\lambda}+\mu)
$$

for $\tilde{\lambda} \in \mathbb{Z}^{I} / \mathbb{Z}^{J}, \mu \in \mathbb{Z}^{J}, \tilde{\lambda}, \mu \gg 0$ where we use the canonical isomorphism $\mathbb{Z}^{I}=$ $\mathbb{Z}^{I} / \mathbb{Z}^{J} \oplus \mathbb{Z}^{J}$

The following two statements follow easily from the definitions.

Suppose that an analytic unimodular $F$-group $H$ acts freely on $\mathrm{W}$ preserving irreducible components of $S=\mathrm{W} \backslash D$. Then for any strata $S_{I}^{0}$ we have a natural action of $H$ on $N_{I}$ and on $N_{I}^{0}$. We denote by $\pi: \mathrm{W} \rightarrow \overline{\mathrm{W}}:=\mathrm{W} / H$ the natural projection. Then $\pi$ defines projection $\pi_{I}: N_{I} \rightarrow \bar{N}_{I}$ where $\bar{N}_{I}$ is the normal bundle to $\bar{S}_{I}^{0}:=S_{I}^{0} / H$ in $\overline{\mathrm{W}}$. We define push-forwards $\pi_{\star}: \mathcal{S}(\mathrm{W}) \rightarrow \mathcal{S}(\overline{\mathrm{W}}), \pi_{I \star}: \mathcal{S}\left(N_{I}\right) \rightarrow$ $\mathcal{S}\left(\bar{N}_{I}\right)$ as integration along $H$. 
Claim 3.8. The asymptotic embeddings

$$
j_{I}: \mathcal{S}\left(N_{I}\right) \rightarrow \mathcal{S}(U)_{\text {as }}(I), \quad \bar{j}_{I}: \mathcal{S}\left(\bar{N}_{I}\right) \rightarrow \mathcal{S}(\bar{U})_{\text {as }}(I)
$$

are compatible with push-forwards $\pi_{\star}, \pi_{I_{\star}}$.

Claim 3.9. Let $Z \subset \mathrm{W}$ be a locally closed smooth subvariety which intersects each of $S_{I}$ transversely. Then the asymptotic embeddings for $\mathrm{W}$ and $Z$ are compatible under the restriction map.

Let $U_{I} \subset \mathrm{W}, I \subset \Sigma$ be open neighborhoods of $S_{I}$ and let

$$
\tau_{I}: U_{I} \rightarrow N_{I}, I \subset \Sigma, \quad \tau_{I}^{J}: \tilde{N}_{J}^{I} \rightarrow r_{J}^{\star}\left(N_{J}^{I}\right), J \subset I
$$

be a family of admissible maps (cf. Claim 3.1 b) where $\tilde{N}_{J}^{I} \subset N_{J}$ are open neighborhoods of $r_{J}^{-1}\left(S_{I}\right)$.

Definition 3.10. We say that a family $\left(U_{I}, \tau_{I}, \tau_{I}^{J}\right)$ is admissible if for any pair $\emptyset \subset J \subset I \subset \Sigma$ there exists an open neighborhood $U^{\prime} \subset U$ of $S_{I}$ in W such that the restriction of $\tau_{I}^{J} \circ \tau_{J}$ on $U^{\prime}$ coincides with $\tau_{I}$.

The following result is easy to prove by induction on $|\Sigma|$.

Claim 3.11. An admissible family exists for any $\mathrm{W}, D$ as above.

3.1. The almost homogeneous case. In this section we suppose that the variety W (hence also $S=\mathrm{W} \backslash D$ ) is compact, that an analytic unimodular $F$-group $H$ acts on W preserving irreducible components of $S$ and that the action on $S_{I}^{0}$ is transitive for all $I \subset \Sigma$. Let $K \subset H$ be an open compact subgroup and let $\left(U_{I}, \tau_{I}, \tau_{I}^{J}\right)$ be an admissible family as in Definition 3.10 .

Lemma 3.12. For any $s \in S_{I}$ and a compact subset $C$ of $H$ there exists an open neighborhood $U$ of $s$ in $\mathrm{W}$ such that $\tau_{I}(c K u)=c K \tau_{I}(u)$ for all $c \in C, u \in U$.

Proof. We can assume without loss of generality that $U=c K$ for some $c \in H$. Then the statement follows by applying Lemma 3.4 to $\tau_{I 1}=\tau_{I}, \tau_{I 2}=\tau_{I} \circ \mathrm{c}$ and $f=\delta_{K u}$ for some $u \in U$.

Proposition 3.13. For any compact subset $C$ of $H$ there exists an open neighborhood $V$ of $S$ in $\mathrm{W}$, such that $V \subset \cup U_{I}$ and $\tau_{I}(h K v)=h K \tau_{I}(v)$ for all $h \in C$, $v \in V, I \subset \Sigma$.

Proof. By induction in $n$ we construct an open set $V_{n}$ such that the required equality holds for all $I$ with $|\Sigma-I| \leq n$.

Consider first the case $I=\Sigma$. Then the variety $S_{I}^{0}$ is compact and therefore is a finite union of $K$-orbits. Therefore there exist a $K$-invariant open neighborhood $U$ of $S_{I}$ in $N_{I}$ and a finite number of points $u_{a} \in U, a \in A$ such that any $u \in U \cap D$ can be written in the form

$$
u=k T_{\lambda} u_{a}, \quad a \in A, k \in K, \lambda \in \mathbb{Z}^{I} .
$$

The statement now follows from Lemma 3.4 .

Assume now that the statement is known for all $J \subset \Sigma$ containing $I$. For any $j \in$ $\Sigma-I$ we choose an open neighborhood $\tilde{N}_{I \cup j}^{I}$ of $r_{I \cup j}^{-1}\left(S_{I}\right)$ satisfying the requirement of Definition 3.10. Then there exist a $K$-invariant open neighborhood $U$ of $S_{I}$ in $N_{I}$ 
and a finite number of points $u_{a} \in U, a \in A$ such that any $u \in U \cap D-\bigcup_{j \in \Sigma-I} \tilde{N}_{I \cup j}^{I}$ can be written in the form

$$
u=k T_{\lambda} u_{a}, \quad a \in A, k \in K, \lambda \in \mathbb{Z}^{I} .
$$

The statement follows now from Lemma 3.4 and the definition of the admissible family.

3.2. The singular case. We now drop the assumption that the analytic variety $\mathrm{W}$ is smooth and the divisor $S \subset \mathrm{W}$ is a divisor with normal crossing. Instead we assume that each stratum $S_{I}$ is well approximated in the sense of the Appendix. The definition of an admissible map applicable to this case is given in the Appendix. Other definitions and results of the present section carry over to this case mutatis mutandis.

\section{An Algebraic Lemma}

Let $A$ be a Noetherian $\mathbb{C}$-algebra, $M$ a finitely generated $A$-module, $T$ an automorphism of $M$ as an $A$-module.

Definition 4.1. Let $M, T$ be as above and $M^{0} \subset M$ be a $T^{-1}$-invariant $\mathbb{C}$ subspace such that $M=\bigcup_{r>0} T^{r} M^{0}$. Let $N$ be an $A$-module. A $\mathbb{C}$-linear map $a: M^{0} \rightarrow N$ will be called $A$-compatible if for any $m \in M, h \in A$ there exists an integer $N(m, h)>0$ such that $h a\left(T^{-n} m\right)=a\left(h T^{-n} m\right)$ for $n>N(m, h)$.

Lemma 4.2. For any $A$-compatible $\mathbb{C}$-linear map a: $M^{0} \rightarrow N$ there exists a unique $A$-morphism $\tilde{a}: M \rightarrow N$ such that for any $m \in M$ we have $\tilde{a}\left(T^{-n} m\right)=a\left(T^{-n} m\right)$ for $n \gg 0$.

Proof. Fix a finite set of generators $m_{i}$ of $M$ over $A$. For some $n>0$ we have $T^{-n} m_{i} \in M^{0}$. If $\tilde{a}$ satisfies the assumption of the lemma, then for large $n$ we have: $\tilde{a}\left(T^{-n} m_{i}\right)=a\left(T^{-n} m_{i}\right)$; however, for any $n$ the elements $T^{-n} m_{i}$ generate $M$, so uniqueness is clear.

We now proceed to show existence. Assume without loss of generality that the generators $m_{i}$ lie in $M_{0}$. Let $\tilde{M} \cong A^{s}$ be the free $A$ module with free generators denoted $\tilde{m}_{1}, \ldots \tilde{m}_{s}$, and $\Pi: \tilde{M} \rightarrow M$ be the surjection sending $\tilde{m}_{i}$ to $m_{i}$. Let $\tau: \tilde{M} \rightarrow \tilde{M}$ be an endomorphism lifting $T^{-1}$.

For $n \geq 0$ define a homomorphism $\alpha_{n}: \tilde{M} \rightarrow N$ by requiring that $\alpha_{n}\left(\tilde{m}_{i}\right)=$ $a\left(T^{-n} m_{i}\right)$. Given $x \in \tilde{M}$ the $A$-compatibility condition implies that

$$
\alpha_{n}(x)=a\left(T^{-n} \Pi(x)\right)
$$

for large $n$.

Applying this to $x_{i}=\tau\left(\tilde{m}_{i}\right)$ we conclude that there exists $n_{0}>0$ such that $\alpha_{n+1}=\alpha_{n} \circ \tau$ when $n \geq n_{0}$. Set $\alpha=\alpha_{n_{0}}$.

Given $x \in \tilde{M}$ we have

$$
\alpha\left(\tau^{n} x\right)=a\left(T^{-n-n_{0}} \Pi(x)\right)
$$

for large $n$.

Since $\tau$ induces an invertible map on $\tilde{M} / \operatorname{Ker}(\Pi)=M$, it follows that for $n>0$ we have: $\Pi(x)=0 \Longleftrightarrow \Pi \tau^{n}(x)=0$, hence $\tau^{n}: \operatorname{Ker}(\Pi) \rightarrow\left(\operatorname{Ker}(\Pi) \cap \operatorname{Im}\left(\tau^{n}\right)\right)$.

Since $A$ is Noetherian, $\operatorname{Ker}(\Pi)$ is finitely generated. Fix a finite set of generators, and pick $n$ for which (4.1) holds when $x$ belongs to this set of generators. Then $\alpha$ vanishes on $\operatorname{Ker}(\Pi) \cap \operatorname{Im}\left(\tau^{n}\right)=\tau^{n}(\operatorname{Ker}(\Pi))$. Since $\left.\Pi\right|_{\operatorname{Im}\left(\tau^{n}\right)}$ is onto, the 
homomorphism $\left.\alpha\right|_{\operatorname{Im}\left(\tau^{n}\right)}$ factors through a homomorphism $\tilde{a}^{\prime}: M \rightarrow N$. Now set $\tilde{a}=\tilde{a}^{\prime} \circ T^{n_{0}}$, then (4.1) implies that $\tilde{a}$ satisfies the required condition.

\section{Construction and properties of the maps $B_{I}$}

We modify the meaning of notation: from now on $G, B, \mathcal{B}, X$, etc. will denote the groups/spaces of $F$-points of algebraic groups or varieties considered in section 2 these are equipped with the $p$-adic topology.

We apply the construction of section 3 to $W=\bar{G}$ being the wonderful compactification, with $D=G$. If $G$ is adjoint this is a particular case of the situation introduced at the beginning of section 3 otherwise, one should apply considerations of subsection 3.2. We fix an admissible system $\tau_{I}$.

5.1. Definition of $\mathrm{B}_{I}$. Let $K \subset G$ be a nice (in the sense of [2]) compact open subgroup and let

$$
\mathcal{H}=\mathcal{S}(G)^{K \times K}, \quad \mathcal{H}_{L}=\mathcal{S}(L)^{K \cap L \times K \cap L}
$$

be the corresponding Hecke algebras. We use an arbitrarily chosen Haar measure on $G$ to identify $\mathcal{H}$ with the space of compactly supported $K$ bi-invariant measures on $G$, thereby endowing it with an algebra structure and the same applies to $\mathcal{H}_{L}$.

It is clear that $\mathcal{S}\left(X_{I}\right)=i_{I} \circ r_{I}^{-}(\mathcal{S}(G))$. We set $M:=\mathcal{S}\left(K \backslash X_{I} / K\right)$. We define $T \in \operatorname{Aut}(M)$ by $T=T^{(1, \ldots, 1)}$ (notations of Definition 3.3). We also fix a finite subset $\left\{h_{q}\right\}, q \in Q$ in $\mathcal{H}^{\otimes 2}$ which generates $\mathcal{H}^{\otimes 2}$ as a ring.

Let $C:=\bigcup_{q \in Q} \operatorname{supp}\left(h_{q}\right) \subset G \times G$, let $V \subset N_{I}$ be an open neighborhood of $S_{I}^{0}$ as in Proposition 3.13 and set

$$
M^{0}:=\left\{f \in M^{K} \mid \operatorname{supp}(f) \subset V\right\} .
$$

Let $N=\mathcal{H}$, and $a: M^{0} \rightarrow N$ be the map coming from the admissible system $\left(\tau_{I}\right)$.

Proposition 5.1. The above data of $A=\mathcal{H}^{\otimes 2}$ modules $M, N$, the subspace $M^{0} \subset$ $M$ and automorphism $T$ satisfy conditions of Lemma 4.2 .

Proof. Noetherian property of $\mathcal{H}^{\otimes 2}$ and finite generation of $M=\mathcal{S}\left(K \backslash X_{I} / K\right)$ follows from [2] (see in particular Remark 3.11 in loc. cit.). The linear map $a$ : $M^{0} \rightarrow N$ satisfies the compatibility condition of Definition 4.1 when $h=h_{q}$ by Proposition 3.13. It follows that it also holds when $h=h_{q_{1}} \cdots h_{q_{n}}$ for any $q_{1}, \ldots, q_{n} \in Q$, hence it holds for any $h \in \mathcal{H}^{\otimes 2}$.

Now Lemma 4.2 implies our main existence result:

Corollary 5.2. There exists unique $\mathcal{H}^{\otimes 2}$-covariant map $\mathrm{B}_{I}^{K}: M^{K} \rightarrow \mathcal{H}$ such that $\mathrm{B}_{I}\left(T^{-n} f\right)=\tau_{I}^{*}\left(T^{-n} f\right)$ for all $f \in M^{0}, n \gg 0$.

Definition 5.3. We denote by $\mathrm{B}_{I}: \mathcal{S}\left(X_{I}\right) \rightarrow \mathcal{S}(G)$ the linear operator whose restriction to $\mathcal{S}\left(K \backslash X_{I} / K\right)$ is equal to $\mathrm{B}_{I}^{K}$ for all nice compact open subgroups $K \subset G$.

We will sometimes refer to $B_{I}$ as Bernstein's map. 
5.2. The induced map on $U_{P}$ coinvariants. Fix a parabolic $P=P_{I}$. Consider the projection $X_{I} \rightarrow G / P_{I} \times G / P_{I}^{-} \stackrel{p r_{2}}{\longrightarrow} G / P_{I}^{-}$. Let $X_{I}^{0}$ be the preimage of the open $U_{P}$ orbit on $G / P_{I}^{-}$under the composed map. Notice that the right action of $U_{P}$ on $X_{I}^{0}$ is free and $X_{I}^{0} / U_{P} \cong G / U_{P}$ canonically. It follows that $\mathcal{S}\left(X_{I}^{0}\right)_{U_{P}}=\mathcal{S}\left(G / U_{P}\right)$.

Likewise, let ${ }^{0} X_{I}$ be the preimage of the open $U_{P}^{-}$orbit under the composition $X_{I} \rightarrow G / P_{I} \times G / P_{I}^{-} \stackrel{p r_{1}}{\longrightarrow} G / P_{I}$. Then the $U_{P-}$ action on ${ }^{0} X_{I}$ is free and ${ }^{0} X_{I} / U_{P}^{-} \cong$ $G / U_{P^{-}}$canonically, hence $\mathcal{S}\left({ }^{0} X_{I}\right)_{U_{P^{-}}}=\mathcal{S}\left(U_{P^{-}} \backslash G\right)$.

Proposition 5.4. a) The composed map

$$
\mathcal{S}\left(G / U_{P}\right)=\mathcal{S}\left(X_{I}^{0}\right)_{U_{P}} \rightarrow \mathcal{S}\left(X_{I}\right)_{U_{P}} \rightarrow \mathcal{S}(G)_{U_{P}}=\mathcal{S}\left(G / U_{P}\right)
$$

where the first arrow is induced by the open embedding and the second one by the map $\mathrm{B}_{I}$, is equal to identity.

b) The composed map

$$
\mathcal{S}\left(G / U_{P^{-}}\right)=\mathcal{S}\left({ }^{0} X_{I}\right)_{U_{P^{-}}} \rightarrow \mathcal{S}\left(X_{I}\right)_{U_{P^{-}}} \rightarrow \mathcal{S}(G)_{U_{P^{-}}}=\mathcal{S}\left(G / U_{P^{-}}\right)
$$

where the first arrow is induced by the open embedding and the second one by the map $\mathrm{B}_{I}$, is equal to identity.

Proof. Consider first the composed map in (a). In view of Claim 3.8 and Claim 2.13 this map restricted to $K$-bi-invariant functions on a neighborhood of $G / U_{I} Z_{I} \subset$ $\overline{G / U_{I}}$ comes from an admissible map $\overline{G / U_{I}} \supset V \rightarrow U \subset N_{\overline{G / U_{I}}}\left(G / U_{I} Z_{I}\right)$. However, it is easy to see that there exists an algebraic isomorphism

$$
N_{\overline{G / U_{I}}}\left(G /\left(U_{I} Z_{I}\right)\right) \cong \overline{G / U_{I}}
$$

such that the composed map

$$
X_{I}^{0} \hookrightarrow N_{\bar{G}}\left(\bar{G}^{0}(I)\right) \rightarrow N_{\overline{G / U_{I}}}\left(G /\left(U_{I} Z_{I}\right)\right) \cong \overline{G / U_{I}}
$$

coincides with the projection $X_{I}^{0} \rightarrow G / U_{I} \subset \overline{G / U_{I}}$. (Here the second arrow in the displayed formula is the differential of the projection $\left.\bar{G}^{0}(I) \rightarrow \overline{G / U_{I}}\right)$.

Thus identity map $\mathcal{S}(G / U) \rightarrow \mathcal{S}(G / U)$ comes from an admissible map for $\overline{G / U_{I}}$. It follows that the map in part (a) restricted to $K$-biinvariant functions on some neighborhood of the zero section $G /\left(U_{I} Z_{I}\right)$ equals identity. Since the map is $G$ equivariant, it is equal to identity on all functions. This proves (a), part (b) is similar.

5.3. The map $\mathrm{B}_{I}$ and $K_{0}$ cosets. Let $K_{0} \subset G$ be the standard open compact, i.e., $K_{0}$ is the group of $O$ points in the standard $O$ form of $G$ (recall that $G$ is assumed to be split).

Recall the canonical bijections $K_{0} \backslash G / K_{0}=X_{*}(\mathbb{T}) / W=X_{*}(\mathbb{T})^{+}, K_{0} \backslash X_{I} / K_{0}=$ $X_{*}(\mathbb{T})_{W_{I}}=X_{*}(\mathbb{T})_{I}^{+}$, where $W_{I}$ is the corresponding parabolic Weyl group and $X_{*}(\mathbb{T})_{I}^{+}$is the set of coweights positive on simple roots in the Levi. These are constructed by fixing a maximal torus $T \subset G$ and a Borel subgroup $B$ containing $T$. Without loss of generality we can assume that the parabolic $P_{I}, I \subset \Sigma$ contains $B$. Thus we get an embedding $T \rightarrow\left(G / U_{I} \times G / U_{I}^{-}\right) / L_{I}=X_{I}$. Let $\iota_{I}$ be the composition of this embedding with the embedding $X_{*}(T) \rightarrow T$ sending a cocharacter $\nu$ to $\nu(\pi)$, where $\pi$ is a uniformizer. For $\lambda \in X_{*}(\mathbb{T})^{+}, \nu \in X_{*}(\mathbb{T})_{I}^{+}$the subsets $G_{\lambda}=K_{0} \iota(\lambda) K_{0},\left(X_{I}\right)_{\nu}=K_{0} \iota_{I}(\nu) K_{0}$ do not depend on the auxiliary choices (here we abbreviated $\left.\iota=\iota_{\emptyset}: \Lambda \rightarrow G=X_{\emptyset}\right)$. 
Assume that $K$ is normalized by the maximal compact subgroup $K_{0}$. Then $K_{0} \times K_{0}$ acts on $K \backslash G_{\lambda} / K$. It is easy to see that if $\lambda$ is such that $(\lambda, \alpha) \gg 0$ for any root $\alpha$ in the radical of $P$, then the stabilizer of the point $K \iota(\lambda) K$ in $K_{0}^{2}$ contains $K_{0}^{+} \times K_{0}^{-}$, where $K_{0}^{+}=K_{0} \cap \operatorname{Rad}\left(P_{I}\right), K_{0}^{-}=K_{0} \cap \operatorname{Rad}\left(P_{I}^{-}\right)$. Since the group $K$ admits a triangular decomposition: $K=K_{+} K_{L} K_{-}$where $K_{L}=L \cap K$, $K_{+}=K \cap \operatorname{Rad}\left(P_{I}\right), K_{-}=K \cap \operatorname{Rad}\left(P_{I}^{-}\right)$, it is easy to see that this stabilizer coincides with the stabilizer of the corresponding point in $K \backslash\left(X_{I}\right)_{\lambda} / K$. Therefore there is a natural $K_{0}^{2}$-equivariant bijection $\Psi_{P}: K \backslash\left(X_{I}\right)_{\lambda} / K \stackrel{\longrightarrow}{\longrightarrow} K \backslash G_{\lambda} / K$.

Lemma 5.5. Fix a congruence subgroup $K \subsetneq K_{0}$. There exists $N>0$ such that for any $\lambda \in \Lambda^{+}$satisfying $(\lambda, \alpha)>N$ for all roots $\alpha$ in the radical of $U_{I} \subset P_{I}$, the following holds:

a) The map $\mathrm{B}_{I}$ sends $\mathcal{S}^{K \times K}\left(\left(X_{I}\right)_{\lambda}\right)$ to $\mathcal{S}^{K \times K}\left(G_{\lambda}\right)$ and induces an isomorphism $\mathrm{B}_{I}: \mathcal{S}^{K \times K}\left(\left(X_{I}\right)_{\lambda}\right) \longrightarrow \mathcal{S}^{K \times K}\left(G_{\lambda}\right)$.

b) Moreover, the map $\left.\mathrm{B}_{I}\right|_{\mathcal{S}\left(X_{I}\right)_{\lambda}}$ coincides with the map induced by bijection $\Psi_{P}$.

Proof. For an integer $N$ let $\left(X_{I}\right)_{N}$ be the union of $\left(X_{I}\right)_{\lambda}$ over $\lambda$ satisfying the condition of the lemma. These sets form a system of fundamental neighborhoods of the closure $S_{I}$ of the corresponding stratum. For each stratum $S_{J}$ in the closure choose a neighborhood $V_{J}$ of $S_{J}$ in $G$. Without loss of generality we can assume that for each $J$ and an admissible system of maps $\phi_{J}: V_{J} \rightarrow X_{J}$ the map $\left.\mathrm{B}_{J}\right|_{\mathcal{S}\left(\phi_{J}\left(V_{J}\right)\right)}$ coincides with $\phi_{J}^{*}$. We can also assume that $V_{J} \subset V$ where $V$ is as in Proposition 3.13 for $C=K_{0}^{2}$. Thus the maps $\phi_{J}^{*}$ on $K$-biinvariant functions are $K_{0}^{2}$ equivariant.

Fix $T \subset B$ as in subsection 5.3 . The choice of Borel subgroup $B$ defines an isomorphism $T \leftrightharpoons \mathbb{T}$. In view of Claim $2.17 \mathrm{~h})$, the resulting embedding $\left(\mathbb{G}_{m}\right)^{\Sigma} \rightarrow G$ extends to an embedding $\overline{\mathbb{T}} \rightarrow \bar{G}$. Moreover, the intersection of its image with any stratum $G_{I}$ (notations of subsection 2.4) is a single $T$-orbit. It is easy to see that there is an admissible system for $\overline{\mathbb{T}}$ where each map is a $T$-equivariant open embedding sending $\iota_{I}(\nu)$ to $\iota_{J}(\nu)$ for $I \subset J$; here we use that the image of $\iota_{I}$ lies in

$$
N_{\left(\mathbb{A}^{1}\right)^{\Sigma}}\left(\left(\mathbb{A}^{1}\right)^{\Sigma} \cap \bar{G}_{I}\right) \subset N_{\bar{G}}\left(\bar{G}_{I}\right) \supset X_{I} .
$$

It also follows from the definitions that under the closed embedding this admissible system is compatible with the one for $\bar{G}$. This yields part (b) of the lemma, which clearly implies part (a).

\section{SeCOnd ADJointness}

6.1. Basic notations. Notice that the spaces $X_{I}, G$ carry $G^{2}$ invariant measures (though $Y_{I}$ does not) and $G / U_{I}$ carries a $G$-invariant measure. We fix such measures and identify the space $\mathcal{S}$ of locally constant compactly supported functions on $X_{I}$, $G, G / U_{I}$ with the space of locally constant compactly supported measures.

We let $\mathcal{M}\left(Y_{I}\right)$ be the space of locally constant compactly supported sections of the locally constant sheaf $\mu_{p r_{2}}$ of fiberwise measures with respect to the second projection $p r_{2}: Y_{I} \rightarrow G / P_{I}$. One can interpret elements $\phi \in \mathcal{M}\left(Y_{I}\right)$ as operators $\hat{\phi}: \mathcal{S}\left(G / U_{I}\right) \rightarrow \mathcal{S}\left(G / U_{I}\right)$ where

$$
\hat{\phi}(\psi)(\bar{g}):=\int_{\bar{g}^{\prime} \in G / U_{I}} \phi\left(\bar{g}, \bar{g}^{\prime}\right) \psi\left(\bar{g}^{\prime}\right) d g^{\prime}, \psi \in \mathcal{S}\left(G / U_{I}\right) .
$$

Definition 6.1. We define the action map

$$
\mathrm{A}_{I}: \mathcal{S}(G) \rightarrow \mathcal{M}\left(Y_{I}\right), \quad f \mapsto \pi_{2 *} \pi_{1}^{*}(f),
$$


where $\pi_{1}:(G \times G) / P_{I} \rightarrow G, \pi_{1}\left(g_{1}, g_{2}\right)=g_{1} g_{2}^{-1} ; \pi_{2}:(G \times G) / P_{I} \rightarrow Y_{I}$, $\pi_{2}\left(\left(g_{1}, g_{2}\right) P_{I}\right)=\left(g_{1}, g_{2}\right)$ and $\pi_{2 *}$ is the fiberwise integration map $\mathcal{S}((G \times G) / P) \rightarrow$ $\mathcal{M}\left(Y_{I}\right)$; here we use the observation that the tensor product of the sheaf $\mu_{\pi_{2}}$ of fiberwise measures for the map $(G \times G) / P_{I} \rightarrow Y_{I}$ and $\pi_{2}^{*}\left(\mu_{p r_{2}}\right)$ is canonically trivialized.

Remark 6.2. In terms of the interpretation of elements in $\mathcal{M}\left(Y_{I}\right)$ as operators, the map $A$ is easily seen to correspond to the action of the Hecke algebra on the universal principal series $\mathcal{S}\left(G / U_{I}\right)$.

Let $P=L U_{P} \subset G$ be a parabolic subgroup and $P^{-}=L U_{P}^{-}$be the opposite parabolic subgroup. Let $i_{I}, r_{I}$ be, respectively, the normalized parabolic induction and the normalized Jacquet functors with respect to $P_{I}$ and $i_{I}^{-}, r_{I}^{-}$be those with respect to $P_{I}^{-}$.

6.2. The adjunction maps. For a smooth $L_{I}$-module $N$ one defines canonical morphisms

$$
\text { can }: r_{I} i_{I}(N) \rightarrow N, \text { Can }: N \rightarrow r_{I}^{-} i_{I}(N) \text {. }
$$

To recall these observe that elements of the space of induced representations are $N$-valued functions on $G$, the map can comes from restriction of functions to the closed subset $P_{I} \subset G$, while $C a n$ comes from push-forward of compactly supported functions from the open subset $U_{P_{I}^{-}} P_{I} \subset G$. Using these canonical morphisms one can define for any smooth $G$-module $M$ and a smooth $L_{I}$-module $N$ the following maps:

$$
\begin{gathered}
\operatorname{Hom}\left(M, i_{I}(N)\right) \rightarrow \operatorname{Hom}\left(r_{I}(M), N\right), \quad \phi \mapsto \operatorname{can} \circ r(\Phi), \\
\operatorname{Hom}\left(i_{I}(N), M\right) \rightarrow \operatorname{Hom}\left(N, r_{I}\left(M^{-}\right), N\right), \quad \psi \mapsto r_{I}^{-}(\psi) \circ \text { Can. }
\end{gathered}
$$

Frobenius adjointness amounts to the morphism (6.1) being an isomorphism. This is a standard fact, one of possible proofs is as follows. It suffices to define $\alpha: I d \rightarrow i_{I} r_{I}$ so that the compositions $i_{I} \rightarrow i_{I} \circ r_{I} \circ i_{I} \rightarrow i_{I}, r_{I} \rightarrow r_{I} \circ i_{I} \circ r_{I} \rightarrow r_{I}$ are equal to identity. Here the arrows $i_{I} \rightarrow i_{I} \circ r_{I} \circ i_{I}, r_{I} \rightarrow r_{I} \circ i_{I} \circ r_{I}$ come from $\alpha$, and the arrows $i_{I} \circ r_{I} \circ i_{I} \rightarrow i_{I}, r_{I} \circ i_{I} \circ r_{I} \rightarrow r_{I}$ come from 6.1).

The morphism $\alpha$ comes from the map $\mathrm{A}_{I}: \mathcal{S}(G) \rightarrow \mathcal{M}\left(Y_{I}\right)$, since it is easy to see that $\mathcal{M}\left(Y_{I}\right) \otimes_{\mathcal{H}} M=i_{I} r_{I}(M)$, and it is obvious that $\mathcal{S}(G) \otimes_{\mathcal{H}} M=M$. The compatibilites are easy to check.

6.3. Second adjointness. We apply a similar strategy to show that (6.2) is also an isomorphism.

We have isomorphisms $i_{I} \circ r_{I}^{-}(M) \cong \mathcal{S}(X) \otimes_{G} M$ and $M \cong \mathcal{S}(G) \otimes_{G} M$. Thus the map $\mathrm{B}_{I}$ yields for every $M \in S m(G)$ a map

$$
\mathrm{B}_{I}(M): i_{I} \circ r_{I}^{-}(M) \rightarrow M .
$$

In other words Bernstein's morphism $\mathrm{B}_{I}$ defines a morphism $\beta: i_{I} \circ r_{I}^{-} \rightarrow I d$. Consider the compositions:

$$
\nu_{I}: i_{I} \stackrel{I d \otimes C a n}{\longrightarrow} i_{I} \circ r_{I}^{-} \circ i_{I} \stackrel{\beta \otimes I d}{\longrightarrow} i_{I}
$$

and

$$
\tau_{I}: r_{I}^{-} \stackrel{C a n \otimes I d}{\longrightarrow} r_{I}^{-} \circ i_{I} \circ r^{-} \stackrel{I d \otimes \beta}{\longrightarrow} r^{-}{ }_{I}
$$


Theorem 6.3. a) The morphisms $\nu_{I}$ and $\tau_{I}$ are the identity morphisms.

b) The map (6.2) is an isomorphism. In particular, $i_{I}$ is the left adjoint of $r_{I}^{-}$.

Proof. (b) follows from (a) by a standard argument. To check (a) observe that the maps of functors $\nu_{I}, \tau_{I}$ come from the maps of bimodules considered, respectively, in parts (a) and (b) of Proposition 5.4. Thus part (a) of the theorem follows from that proposition.

\section{BERNSTEIN'S MAP AND INTERTWINING OPERATORS}

In this section we formulate some properties of the maps introduced above and state a result which says that the maps $A$ and $B$ are related by the intertwining operator. The proof occupies the next two sections.

To simplify notation we only treat the case of the spaces $X, Y$ attached to the Borel subgroup, we expect that similar results can be proved in a similar way for spaces $X_{I}, Y_{I}$.

Let $\mathcal{S}^{\prime}(G), \mathcal{S}^{\prime}(X)$ denote the space of functions which are invariant under $K \times K$ for some open compact subgroup $K$ (but are not necessarily compactly supported). Our choice of Haar measure defines a pairing between $\mathcal{S}(X)$ (respectively, $\mathcal{S}(G)$ ) and $\mathcal{S}^{\prime}(X)$ (respectively, $\mathcal{S}^{\prime}(G)$ ), identifying $\mathcal{S}^{\prime}(X), \mathcal{S}^{\prime}(G)$ with smooth dual of $\mathcal{S}(X), \mathcal{S}(G)$ respectively. We let $\mathrm{B}^{\star}: \mathcal{S}^{\prime}(G) \rightarrow \mathcal{S}^{\prime}(X)$ denote the operator dual to B.

7.1. Bounded supports. Recall that $\bar{X}_{\text {aff }}$ denotes (the space of $F$-points of) the affine closure of the quasi-affine algebraic variety $X$. Let us say that a subset $C \subset X$ is bounded if the closure of $C$ in $\bar{X}_{\text {aff }}$ is compact.

Let $\mathcal{S}_{b}(X)$ denote the space of locally constant functions on $X$ which are supported on a bounded subset in $X$.

Proposition 7.1. For $f \in \mathcal{H}$ the support of $\mathrm{B}^{\star}(f)$ is bounded, i.e.,

$$
\mathrm{B}^{\star}: \mathcal{S}(G) \rightarrow \mathcal{S}_{b}(X)
$$

In view of potential applications we mention a more precise version of this result in a special case; it will not be used in this paper.

Proposition 7.2. The support of $\mathrm{B}^{\star}\left(\delta_{K_{0}}\right)$ is contained in $\bar{X}_{\mathrm{aff}}(O)$.

The proof of Proposition 7.2 appears in section 10.

7.2. Intertwining operators. Fix $w \in W$. Recall the Radon correspondence $\mathfrak{C}_{w}$ appearing in Lemma 2.3. We define the intertwining operator (or Radon transform) $I_{w}: \mathcal{S}(X) \rightarrow \mathcal{M}^{\prime}(Y), I_{w}: f \mapsto p r_{2 *}^{w}\left(p r_{1}^{w}\right)^{*}(f)$ where $p r_{1}^{w}: \mathfrak{C}_{w} \rightarrow X, p r_{2}^{w}: \mathfrak{C}_{w} \rightarrow Y$ are the projections and $\mathcal{M}^{\prime}(Y)$ is the space of locally constant sections of the sheaf $\mu_{p r_{2}}$ (see subsection 6.1).

Proposition 7.3. Let $S \subset X$ be a closed bounded subset. Then the map $\mathrm{pr}_{2}^{w}$ : $\left(p r_{1}^{w}\right)^{-1}(S) \rightarrow Y$ is proper.

Corollary 7.4. The intertwining operator $I_{w}$ naturally extends to a map $I_{w}$ : $\mathcal{S}_{b}(X) \rightarrow \mathcal{M}^{\prime}(Y)$.

Let us say that a subset in $Y$ is $w$-bounded if its closure in $\bar{Y}_{w}$ is compact.

Let $\mathcal{M}_{b}^{w}(Y)$ denote the space of locally constant sections of the sheaf $\mu_{p r_{2}}$ whose support is $w$-bounded. 
Proposition 7.5. a) The map $I_{w}$ sends $\mathcal{S}_{b}(X)$ to $\mathcal{M}_{b}^{w}(Y)$.

b) The $\operatorname{map}_{w}: \mathcal{S}_{b}(X) \rightarrow \mathcal{M}_{b}^{w}(Y)$ is an isomorphism.

\subsection{Main result.}

Theorem 7.6. For any $w \in W$ we have $\mathrm{A}=I_{w} \mathrm{~B}^{\star}$.

Remark 7.7. The latter equality (in the special case $w=1$ ) resembles the result of [3, Corollary 6.2]. More precisely, in loc. cit. one finds an isomorphism of two functors between the categories of $D$-modules. Following a standard analogy between maps of function spaces and functors on the categories of $D$-modules given by "the same" correspondence one gets that one of the two functors considered in loc. cit. is analogous to the map $I_{w}^{-1} \mathrm{~A}$. The other functor in [3] is that of nearby cycles, or specialization. The characterization of $\mathrm{B}$ via cospecialization of functions on an $F$-manifold to normal cone makes it natural to consider $\mathrm{B}^{\star}$ as an analogue of specialization functor between the categories of $D$-modules. It would be interesting to find a precise mathematical statement underlying these heuristic considerations.

The proof of the theorem is given in section 9 .

Corollary 7.8. For any $w \in W$ we have $\mathrm{B}=\mathrm{A}^{\star}\left(I_{w}^{\star}\right)^{-1}$.

\section{Some properties of Radon CORRESPONDENCE}

8.1. Proof of Propositions 7.3, 7.5. Proposition 7.3 follows from Lemma 2.5

Proposition 7.5(a) follows from Proposition 2.6.

In order to prove Proposition 7.5 (b) we show the following more precise result.

Along with $I_{w}$ we will also consider the adjoint operator $I_{w}^{\prime}=p r_{1 *}^{w}\left(p r_{2}^{w}\right)^{*}$ : $\mathcal{M}_{b}(Y) \rightarrow \mathcal{S}^{\prime}(X)$.

Proposition 8.1. There exists an element $\sigma$ in the group algebra of the torus $T$ with the following properties.

i) The element $\sigma$ is a product of elements of the form $\left[\alpha_{i}\right]-c_{i}$ where $c_{i}$ is a constant and $\left[\alpha_{i}\right] \in T$ is a representative of the coset of $T^{0}$ corresponding to a positive coroot.

ii) For $f \in \mathcal{S}(X)$ the element $I_{w}(\sigma(f))$ has compact support.

iii) For $f \in \mathcal{S}(X)$ the element $I_{w}^{\prime} I_{w}(\sigma(f)$ ) (which is well defined by ii) has compact support.

iv) For a character $\chi$ of $T^{0}$ let $\mathcal{S}_{\chi}(X) \subset \mathcal{S}(X)$ be the subspace of $\chi$ semiinvariants with respect to the action of $T^{0}$. For every $\chi$ the restriction of the map $f \mapsto I_{w}^{\prime} I_{w}(\sigma(f))$ to $\mathcal{S}_{\chi}$ equals the action of an element $\sigma_{\chi}$ in the group algebra of $T$, where $\sigma_{\chi}$ is a product of nonzero elements of the form $b_{\chi, i}\left[\alpha_{i}\right]-c_{\chi, i}$ where $\alpha_{i}$ is as in (i).

Proof. Fix a minimal decomposition for elements $w, w^{-1} w_{0}$. This defines a presentation of $I_{w}$ as a composition of $\ell(w)$ simpler correspondences. Thus it suffices to prove a similar statement for each of these simpler correspondences.

This reduces to the following well-known properties of Radon transform.

Claim 8.2 ([10, §II.2.5, II.2.6]). Let $R$ denote Radon transform for functions on the plane, $R(f)(y)=\int f\left(x_{0}+t y\right) d t$ where $x_{0}$ is such that $\langle x, y\rangle=1$ for a fixed skew-symmetric bilinear pairing $\langle$,$\rangle .$

a) If $f \in \mathcal{S}\left(F^{2} \backslash\{0\}\right)$ is such that the integral of $f$ over any line passing through zero vanishes, then $R(f)$ has compact support. 
b) If $\pi \in F$ is the uniformizer and $q$ is the cardinality of the residue field of $F$, then $\pi(f)-q^{-1} f$ satisfies the assumption of (a) for any $f \in \mathcal{S}\left(F^{2} \backslash\{0\}\right)$, where for $t \in F^{\times}$we write $t(f)(x):=f\left(t^{-1} x\right)$.

c) Let $\Phi$ denote Fourier transform for functions on the plane. Then there exists a rational scalar valued function $\gamma$ on the set of multiplicative characters, such that for any $f \in \mathcal{S}\left(F^{2} \backslash\{0\}\right)$ satisfying the assumption of (a) and any multiplicative character $\chi$ in the domain of definition of $\gamma$ we have

$$
\gamma(\chi) \cdot \overline{R(f)}_{\chi}=\overline{\Phi(f)}_{\chi} .
$$

Here for $\phi \in \mathcal{S}\left(F^{2} \backslash\{0\}\right)$ we let $\bar{\phi}_{\chi}$ denote the image of $\phi$ in the space $\mathcal{S}\left(F^{2} \backslash\{0\}\right)_{\chi}$ of $\chi$-coinvariants with respect to the dilation action of $F^{\times}$.

We also have $\Phi^{2}=I d$.

Remark 8.3. The above proof does not apply in the case when a Borel subgroup is replaced by a parabolic one. It is possible to give an alternative proof admitting such a generalization.

8.1.1. Proof of Proposition $7.5(b)$. In view of Proposition $8.1 I_{w}$ induces an isomorphism between the spaces $\mathcal{S}(X)$ and $\mathcal{M}(Y)$ tensored with localization of the group algebra of the torus $T$ by some elements of the form $\left[\alpha_{i}\right]-c_{i}$ where $\alpha_{i}, c_{i}$ are as in Proposition 8.1. The subset $\left\{\alpha_{i}^{n} \mid n \geq 0\right\} \subset T$ is bounded, which shows that for $f \in \mathcal{S}_{b}(X)$ the infinite sum $\sum_{n=0}^{\infty} c_{i}^{-n} \alpha_{i}^{n}(f)$ is a well-defined element of $\mathcal{S}_{b}(X)$. Thus $\left[\alpha_{i}\right]-c_{i}$ acts by an invertible operator on $\mathcal{S}_{b}(X)$, a similar argument shows that it also acts by an invertible operator on $\mathcal{M}_{b}^{w}(Y)$. Proposition 7.5 (b) follows.

The rest of the section is devoted to the proof of Proposition 7.1

\subsection{The Bernstein center and supports.}

8.2.1. The Bernstein center. Let $\mathcal{Z}$ denote the Bernstein center of $G$. Recall $[2$ that $\operatorname{Spec}(\mathcal{Z})$ is the union of connected components, and each component has the form $\operatorname{Spec}\left(\mathcal{Z}_{k}\right)=T_{k} / W_{k}$ where the torus $T_{L_{k}}$ is dual to $L_{k} / L_{k}^{\prime}$ for a Levi subgroup $L_{k}$ in $G$, while $W_{k}$ is a finite group acting on $T_{k}$ (in fact, $W_{k}$ is a subgroup in $T_{L_{k}} \ltimes W_{k}$ where $W_{k}$ is the Weyl group; here we used the standard notation $L^{\prime}=[L, L]$ for the commutant). Thus for every $k$, the summand $\mathcal{Z}_{k}$ is a subalgebra in $\mathbb{C}\left[X_{*}\left(L_{k} / L_{k}^{\prime}\right)\right]$. We have embeddings $X_{*}\left(\mathrm{~A}_{k}\right) \rightarrow X_{*}\left(L_{k} / L_{k}^{\prime}\right), X_{*}\left(\mathrm{~A}_{k}\right) \rightarrow X_{*}(\mathbb{T})$ where $\mathrm{A}_{k}$ is the center of $L_{k}$; the first embedding has a finite index. Thus $X_{*}\left(L_{k} / L_{k}^{\prime}\right) \subset X_{*}(\mathbb{T})_{\mathbb{Q}}:=$ $X_{*}(\mathbb{T}) \otimes \mathbb{Q}$ canonically.

We will need the following property of elements in the Bernstein center $\mathcal{Z}$ which follows directly from the description of $\mathcal{Z}$ in $[2]$.

Claim 8.4. For any element $h \in \mathcal{Z}$ there exists an element $h_{L} \in \mathcal{Z}(L)$ such that the left action of $h$ on $\mathcal{S}\left(G / U_{P}\right)$ coincides with the action of $h_{L}$ coming from the right action of $L$.

8.2.2. Filtrations by support. We define an increasing filtration on $\mathcal{Z}$ as follows. For $\mu \in X_{*}(\mathbb{T})^{+}$set

$$
X_{*}(\mathbb{T})_{\mathbb{Q}}^{\leq \mu}=\left\{\lambda \in X_{*}(\mathbb{T})_{\mathbb{Q}} \mid(w(\lambda), \alpha) \leq(\mu, \alpha) \text { for all simple coroots } \alpha, w \in W\right\},
$$

and define

$$
\left(\mathcal{Z}_{k}\right)_{\leq \mu}=\mathcal{Z}_{k} \cap \mathbb{C}\left[X_{*}(\mathbb{T})_{\mathbb{Q}}^{\leq \mu}\right], \quad \mathcal{Z}_{\leq \mu}=\prod\left(\mathcal{Z}_{k}\right)_{\leq \mu}
$$


This equips $\mathcal{Z}_{k}, \mathcal{Z}$ with filtrations indexed by the partially ordered semi-group of dominant weights. It is easy to see that the Rees ring $\bigoplus_{\mu}\left(\mathcal{Z}_{k}\right)_{\leq \mu}$ is finitely generated for each $k$.

We will need an auxiliary result relating this filtration to a filtration on the Hecke algebra.

Recall notations $K_{0}, G_{\lambda}$, etc., from subsection 5.3 .

We let $G_{\leq \lambda}=\bigcup_{\mu \leq \lambda} G_{\mu}$ and we let $\mathcal{H}_{\leq \lambda}=\left\{h \in \mathcal{H} \mid \operatorname{supp}(h) \subset G_{\leq \lambda}\right\}$; here $\leq$ denotes the standard order on dominant coweights.

Fix a congruence subgroup $K \subsetneq K_{0}$. Thus $K$ is a normal open subgroup in $K_{0}$ and $K$ is nice in the sense of [2]. We change notation and write $\mathcal{H}$ instead of $\mathcal{H}_{K}$ and $\mathcal{H}_{\leq \lambda}$ instead of $\mathcal{H}_{\leq \lambda} \cap \mathcal{H}_{K}$.

Proposition 8.5. There exists $\lambda_{0}$ such that for $\lambda \geq \lambda_{0}$ and any $\mu$ we have

$$
\mathcal{Z}_{\leq \mu} \cdot \mathcal{H}_{\leq \lambda} \subset \mathcal{H}_{\leq \lambda+\mu}
$$

The proof of the proposition is preceded by two auxiliary statements.

The first one is a property of the element $h_{L}$ introduced in Claim 8.4, it is an immediate consequence of characterization of $h_{L}$ formulated in that claim.

Claim 8.6. For $h \in \mathcal{Z}_{\leq \mu}$ let $h_{L}$ be as in Claim 8.4. Then the support $\operatorname{supp}\left(h_{L}\right)$ satisfies the following condition. Let $L_{c} \subset L$ be the subgroup generated by compact subgroups, so that $L / L_{c} \cong X_{*}\left(L / L^{\prime}\right) \subset X_{*}(\mathbb{T})_{\mathbb{Q}}$. The image of $\operatorname{supp}\left(h_{L}\right)$ in $L / L_{c}$ is contained in $X_{*}\left(L / L^{\prime}\right)_{\leq \mu}$.

To state another lemma, note that we can identify the double quotient $K_{0} \backslash X_{I} / K_{0}$ with $X_{*}(\mathbb{T}) / W_{I}=X_{*}(\mathbb{T})_{I}^{+}$, where $W_{I}$ is the corresponding parabolic Weyl group and $X_{*}(\mathbb{T})_{I}^{+}$is the set of coweights positive on simple roots in the Levi subgroup $L_{I}$. For $\nu \in X_{*}(\mathbb{T})_{I}^{+}$we denote by $\left(X_{I}\right)_{\nu}$ the corresponding $K_{0}^{2}$ orbit.

Lemma 8.7. Fix $I \subset \Sigma$. For any $\mu \in X_{*}(\mathbb{T})^{+}$there exists a finite set $S_{\mu}$ of linear combinations of the form $\sum r_{i} \alpha_{i}^{\vee}, i \in I$ such that $\mathcal{Z}_{\leq \mu} \mathcal{S}\left(X_{I}\right)_{\nu}^{K \times K} \subset$ $\sum \mathcal{S}\left(X_{I}\right)_{\eta}, \quad \eta \in \nu+\operatorname{conv}(W(\mu))+S_{\mu}$ where conv is the convex hull.

Proof. We have a fibration $X_{I} \rightarrow G / P_{I}^{-}$with fibers $G / U_{I}$; the set $K_{0} \backslash G /\left(U_{I}\right.$. $\left.K_{0}^{L_{I}}\right)$ maps isomorphically to $K_{0} \backslash X_{I} / K_{0}$. The left action of $\mathcal{H}$ we consider here is fiberwise, thus it suffices to prove a similar statement about the action on $\mathcal{S}\left(G / U_{I}\right)$.

An element $h \in \mathcal{H}(L)$ supported on the preimage of a given $\zeta \in X_{*}\left(L / L^{\prime}\right)$ sends $\mathcal{S}\left(G / U_{I}\right)_{\nu}$ to the sum of $\mathcal{S}\left(G / U_{I}\right)_{\eta}$ where $\eta \in \nu+\zeta+S$ for some finite subset $S=S(h)$ of linear combinations of $\alpha_{i}^{\vee}, i \in I$. Thus the lemma follows from Claim 8.6 .

8.2.3. Proof of Proposition 8.5. Since the Rees ring $\bigoplus_{\mu}\left(\mathcal{Z}_{k}\right)_{\leq \mu}$ is finitely generated for each $k$ and $\mathcal{Z}_{k} \mathcal{H}=0$ for almost all $k$, it suffices to show that for fixed $k, \mu$ and sufficiently large $\lambda$ we have

$$
\left(\mathcal{Z}_{k}\right)_{\leq \mu} \cdot \mathcal{H}_{\leq \lambda} \subset \mathcal{H}_{\leq \lambda+\mu}
$$

Fix $N \in \mathbb{Z}_{>0}$ as in Lemma 5.5. We can and will assume that $N>2 r_{i}$ for $r_{i}$ as in Lemma 8.7, $\mu \in M$. We can and will assume also that $\langle\lambda, \alpha\rangle>2 N$ for any simple root $\alpha$.

Fix $\nu \in X_{*}(\mathbb{T}), \nu \leq \lambda$ and let $f$ be a $K$-bi-invariant function supported on $G_{\nu}$. Let $P=P_{I}$ be the parabolic subgroup such that the simple roots in its Levi are 
exactly those simple roots $\alpha$ for which $(\alpha, \nu) \leq N$. By Lemma [5.5)(a) we have $f=\mathrm{B}_{I}\left(f_{P}\right)$ for some $f_{P} \in \mathcal{S}\left(X_{I}\right)_{\nu}$. Thus for $z \in Z_{\leq \mu}$ we have $z(f)=\mathrm{B}_{I}\left(z f_{P}\right)$. In view of Lemma 8.7, $z\left(f_{P}\right) \in \sum \mathcal{S}\left(X_{I}\right)_{\eta}$ with $\eta \in \nu+\operatorname{conv}(W(\mu))+S_{\mu}$ for a fixed finite set $S_{\mu}$ of linear combination $\sum r_{i} \alpha_{i}$ where $\alpha_{i}$ are coroots of the Levi.

To finish the proof of Proposition 8.5 it suffices to check that $\nu+\sum r_{i} \alpha_{i} \leq \lambda$. To do this, notice that for every simple coroot $\alpha_{i}$ of the Levi and the corresponding fundamental weights $\omega_{i}$ we have

$$
\left(\lambda-\nu, \omega_{i}\right)>\frac{1}{2}\left(\lambda-\nu, \alpha_{i}\right)>\frac{1}{2}(2 N-N)=\frac{1}{2} N>r_{i},
$$

where the first inequality follows from the fact that $\lambda-\nu$ is a sum of positive coroots, while the other ones follow from the assumptions on $N$.

In the next subsection we will need the following consequence of Proposition 8.5 ,

Lemma 8.8. Fix an open compact subgroup $K \subset K_{0}$. There exists a finite subset $S \subset X_{*}(\mathbb{T})$ such that for any $\mu \in X_{*}(\mathbb{T})_{I}^{+}, \phi \in \mathcal{S}\left(X_{I}\right)_{\mu}$ and $\lambda \in X_{*}(\mathbb{T})^{+}$and any $w \in W$ there exist $\psi_{i} \in \mathcal{S}\left(X_{I}\right), h_{i} \in \mathcal{H}$, such that

$$
\phi=\sum h_{i}\left(\psi_{i}\right), \operatorname{supp}\left(h_{i}\right) \subset G_{\leq \lambda}, \operatorname{supp}\left(\psi_{i}\right) \subset\left(X_{I}\right)_{\mu-w(\lambda)+S} .
$$

Proof. The space $\mathcal{S}(X)$ is acted upon by the maximal torus $\mathbb{T}$; for our current purposes it suffices to consider the action of the subgroup $X_{*}(\mathbb{T}) \subset \mathbb{T}$ (where the embedding depends on the choice of a uniformizer $\pi \in F$ ).

It follows from the description of $\mathcal{Z}$ in 2 that the action of an element $s \in$ $\mathbb{C}\left[X_{*}(\mathbb{T})\right]^{W}$ on $\mathcal{S}(X)^{K \times K}$ coincides with an action of some element in $s^{\prime} \in \mathcal{Z}$. Moreover, if $s \in \mathbb{C}\left[X_{*}(\mathbb{T})\right]_{\leq \mu}$ for some $\mu \in X_{*}(\mathbb{T})^{+}$, then $s^{\prime}$ can be chosen in $\mathcal{Z}_{\leq \lambda}$.

It is clear that $\mathcal{S}(X)_{0}$ generates $\mathcal{S}(X)$ as a module over $X_{*}(\mathbb{T})$. Choose a finite dimensional space of generators for $\mathbb{C}\left[X_{*}(\mathbb{T})\right]$ over $\mathbb{C}\left[X_{*}(\mathbb{T})\right]^{W}$, then applying it to $\mathcal{S}(X)_{0}^{K \times K}$ we get a space of generators for $\mathcal{S}(X)^{K \times K}$ over $\mathbb{C}\left[X_{*}(\mathbb{T})\right]^{W}$, let us denote it by $V$. We can also assume without loss of generality that $\mathbb{C}\left[X_{*}(\mathbb{T})\right]_{\leq \lambda} \mathcal{S}(X)_{0} \subset$ $\mathbb{C}\left[X_{*}(\mathbb{T})\right]_{\leq \lambda}^{W} V$ for all $\lambda \in X_{*}(\mathbb{T})^{+}$.

Let us now choose $\lambda_{0}$ as in 8.5 and choose $S$ such that $\mathcal{S}(X)_{S} \supset \mathbb{C}\left[X_{*}(\mathbb{T})\right]_{\leq \lambda_{0}}^{W} \cdot V$. We claim that this $S$ satisfies the condition of Lemma 8.8. Indeed, for $\lambda \geq \lambda_{0}$ we have

$$
\mathcal{S}(X)_{\leq \lambda} \subset \mathbb{C}\left[X_{*}(\mathbb{T})\right]_{\leq \lambda} V=\mathbb{C}\left[X_{*}(\mathbb{T})\right]_{\leq \lambda-\lambda_{0}}^{W} \mathbb{C}\left[X_{*}(\mathbb{T})\right]_{\lambda_{0}}^{W} V \subset \mathcal{H}_{\leq \lambda} \mathcal{S}(X)_{S} .
$$

Here in the last inclusion we used the inclusion $\mathbb{C}\left[X_{*}(\mathbb{T})\right]_{\leq \lambda-\lambda_{0}}^{W} \delta_{K} \subset \mathcal{Z}_{\leq \lambda-\lambda_{0}} \delta_{K} \subset$ $\mathcal{H}_{\leq \lambda}$ which follows from Proposition 8.5

The last ingredient needed in the proof of Proposition 7.1 is the following easy statement.

Claim 8.9. For any $\lambda_{0} \in X_{*}(\mathbb{T})$ the subset $X_{\leq \lambda_{0}}:=\bigcup_{\lambda \leq \lambda_{0}} X_{I, \lambda}$ has compact closure in $\bar{X}_{\text {aff }}$.

8.3. Completion of the proof of Proposition 7.1. Since the space of bounded distributions is clearly invariant under the $G \times G$ action, it suffices to prove the statement for $f=\delta_{K}$.

In view of Claim 8.9 it is enough to show existence of $\lambda_{0} \in X_{*}(\mathbb{T})_{I}^{+}$such that for $\phi \in \mathcal{S}(X)^{K \times K}$ the condition $\operatorname{supp}(\phi) \subset X_{I, \mu}, \mu \not \leq \lambda_{0}$ implies that $\left.\mathrm{B}(\phi)\right|_{1}=0$. 
Assume $\phi \in \mathcal{S}(X)_{\mu}$ and write $\mu=\lambda_{1}-\lambda_{2}$, where $\lambda_{1}, \lambda_{2} \in \Lambda^{+}$. Apply Lemma 8.8 with $w=w_{0}$. We get that $\phi=h(\psi)$, for some $h \in \mathcal{H}_{\leq \lambda_{2}^{\prime}}$ and $\operatorname{supp}(\psi) \subset X_{\lambda_{1}+S}$; here $\lambda_{2}^{\prime}=-w_{0}\left(\lambda_{2}\right)$ is the dual weight. We can and will assume without loss of generality that the pairing of each coweight in $\lambda_{1}+S$ with any fundamental weight is larger than $N$ where $N$ is as in Lemma 5.5. This lemma implies then that $\operatorname{supp}(\mathrm{B}(\psi)) \subset G_{\lambda_{1}+S}$. Thus $\left.f * \mathrm{~B}(\psi)\right|_{1}=0$ for $f \in \mathcal{H}_{\leq \nu}$ unless $\lambda_{1}+\sigma \leq \nu^{\prime}$ for some $\sigma \in S, \nu^{\prime}=-w_{0}(\nu)$. If we choose $\lambda_{0}$ so that $\lambda_{0} \not \leq-\sigma$ for $\sigma \in S$, we get that $\lambda_{2}=\lambda_{1}-\mu \nsupseteq \lambda_{1}+\sigma$, so $\left.h * \mathrm{~B}(\psi)\right|_{1}=0$ since $h \in \mathcal{H}_{\leq \lambda_{2}^{\prime}}$.

\section{Proof of Theorem 7.6}

Recall that $\mathcal{S}_{b}(X), \mathcal{S}_{b}(T)$ denotes the space of locally constant functions on $X$ (respectively, $T$ ) with bounded support; $X_{w_{1}, w_{2}}$ denotes the corresponding $B \times B$ orbit on $X$.

Recall the Jacquet and induction functors $r_{I}=r_{P_{I}}, i_{I}=i_{P_{I}}$ introduced in subsection 6.1 when $P_{I}=B$ is Borel we abbreviate this to $r, i$. We will also abbreviate $r(\mathcal{S}(G))$ to $r(G)$ etc.

Proposition 9.1. The stratification of $X$ by $B \times B$ orbits $X_{w_{1}, w_{2}}$ induces a filtration on $r\left(\mathcal{S}_{b}(X)\right)$ which splits canonically, yielding a canonical isomorphism:

$$
r\left(\mathcal{S}_{b}(X)\right) \cong \bigoplus_{W \times W} \mathcal{S}_{b}(T)
$$

We denote the summand corresponding to $\left(w_{1}, w_{2}\right) \in W \times W$ by $r\left(\mathcal{S}_{b}(X)\right)_{w_{1}, w_{2}}$. The proposition follows from the next lemma which shows that for every $B \times B$ orbit $X_{w_{1}, w_{2}} \subset X$ the map $r\left(\mathcal{S}_{b}(X)\right) \rightarrow \mathcal{S}_{b}(T), f \mapsto\left(p r_{w_{1}, w_{2}}\right)_{*}\left(\left.f\right|_{X_{w_{1}, w_{2}}}\right)$ is well defined; here $p r_{w_{1}, w_{2}}: X_{w_{1}, w_{2}} \rightarrow T \cong X_{w_{1}, w_{2}} /\left(U_{B} \times U_{B}\right)$ is the projection. It is clear that the direct sum of these maps provides an isomorphism (9.1) which splits the filtration.

Lemma 9.2. Let $\beth \subset X$ be a bounded subset. Fix $w_{1}, w_{2} \in W$ and let $X_{w_{1}, w_{2}}$ be the corresponding $B \times B$-orbit. Then the map $\mathrm{pr}_{w_{1}, w_{2}}: \beth \cap X_{w_{1}, w_{2}} \rightarrow T=X_{w_{1}, w_{2}} / U_{B}^{2}$ is proper and its image is bounded.

For every $w_{1}, w_{2} \in W$, every bounded subset in $T$ is the image of $\beth \cap X_{w_{1}, w_{2}}$ under $\mathrm{pr}_{w_{1}, w_{2}}$ for some bounded subset $\beth \subset X$.

Proof. Without loss of generality we can assume that $\beth$ is invariant under an open compact subgroup in $G \times G$. Then properness amounts to compactness of the intersection of $\beth$ with every fiber of the projection $X_{w_{1}, w_{2}} \cap \beth \rightarrow T$, which follows from the fact that the fibers of projection $X_{w_{1}, w_{2}} \rightarrow X_{w_{1}, w_{2}} / U^{2}$ are closed in $\bar{X}_{\text {aff }}$. The latter is a consequence of the Kostant-Rosenlicht Theorem [19, Proposition 2.4.14], since each fiber is an orbit of an affine algebraic group acting on an affine algebraic variety.

In fact, the image of the projection is bounded and the last statement follows respectively from parts b) and a) of Claim 2.17

9.1. The subquotient maps for $\mathrm{B}^{\star}$. In the next statement we use identifications of $T$-torsors $U_{B} \backslash G_{w} / U_{B}$ and $U_{B} \backslash X_{w_{1}, w_{2}} / U_{B}$ where $w_{1} w_{2} w_{0}=w \in W$. Such an identification follows from Corollary 2.16 . 
Lemma 9.3. Suppose that $w=w_{1} w_{2} w_{0}$ and $\ell(w)+\ell\left(w_{0}\right)=\ell\left(w_{1}\right)+\ell\left(w_{2}\right)$. Then the composition $r(G)_{\geq w} \stackrel{r\left(\mathrm{~B}^{\star}\right)}{\longrightarrow} r\left(\mathcal{S}_{b}(X)\right) \rightarrow r\left(\mathcal{S}_{b}(X)\right)_{w_{1}, w_{2}}$ factors through a map $r(G)_{w} \rightarrow r\left(\mathcal{S}_{b}(X)\right)_{w_{1}, w_{2}}$. This map equals the canonical embedding $\mathcal{S}(T) \rightarrow \mathcal{S}_{b}(T)$.

Proof. The composed map in question factors through a map $r(G)_{w} \rightarrow r\left(\mathcal{S}_{b}(X)\right)_{w_{1}, w_{2}}$ because there are no nonzero $T^{2}$ equivariant maps $\left(r(G)_{<w}\right) \rightarrow r\left(\mathcal{S}_{b}(X)\right)_{w_{1}, w_{2}}$.

The statement readily follows from the following formula:

$$
p r_{w_{1}, w_{2} *}\left(\left.\mathrm{~B}^{\star}(f)\right|_{X_{w_{1}, w_{2}}}\right)=\bar{f} ;
$$

here $f \in \mathcal{S}(G)$ is such that $\left.f\right|_{G_{<w}}=0$ and $\bar{f}$ is the image of $f$ in $r(\mathcal{S}(G))_{w}=\mathcal{S}(T)$. Notice that the direct image is well defined since the map

$$
\operatorname{supp}\left(\mathrm{B}^{\star}(f)\right) \cap X_{w_{1}, w_{2}} \rightarrow T=X_{w_{1}, w_{2}} / U_{B}^{2}
$$

is proper in view of Lemma 9.2 and Proposition 7.1

Since the map $p r_{w_{1}, w_{2} *}$ factors through coinvariants with respect to the action of $U_{B} \times U_{B}$, we have $p r_{w_{1}, w_{2} *}\left(\left.\mathrm{~B}^{\star}(f)\right|_{X_{w_{1}, w_{2}}}\right)=F(\bar{f})$ for some $T \times T$ equivariant map $F: \mathcal{S}(T) \rightarrow \mathcal{S}_{b}(T)$. Thus (9.2) would follow if we show that restrictions of both sides to a nonempty open subset $C \subset T$ (which may depend on $K$ but not on $f$ ) coincide. The construction of the map B in section 5] (see Corollary 5.2) makes it clear that for some neighborhood $V_{G}$ of $Z=\mathcal{B} \times \mathcal{B}$ in $\bar{G}$, a neighborhood $V_{X}$ of the zero section in the normal bundle $N_{\bar{G}}(Z)$ and an admissible bijection $\tau: V_{X} \rightarrow V_{G}$ we have $\mathrm{B}(f)=\tau_{*}(f)$ for any $f \in \mathcal{S}(X)^{K \times K}$, $\operatorname{supp}(f) \subset V_{X}$. In view of Claim 3.8 , we will be done if we show that for some $U_{B} \times U_{B}$ invariant subset $V \subset X_{w_{1}, w_{2}}$ we have $V \subset V_{X}$. This follows from the next geometric lemma.

Lemma 9.4. For a representation $M$ of $G$ let $\rho_{M}: X \rightarrow \operatorname{End}(M)$ be the canonical map as in Claim 2.18.

a) Suppose that a subset $\mathfrak{Z} \subset X$ is such that for any $M$ the closure of $\rho_{M}(\mathfrak{Z})$ does not contain zero. Then for any neighborhood $V$ of the zero section in $N_{\bar{G}}(Z) \supset X$ we have $z_{I}^{N}(\mathfrak{Z}) \subset V$ for $N \gg 0$ (where we use the notation of Definition [3.3).

b) Every orbit of the group $U_{B} \times U_{B} \times T_{0}$, where $T_{0}$ is a compact subgroup in the abstract Cartan, satisfies the conditions of part (a).

Proof. (a) follows from Claim 2.18, in view of the following easy observation: given finite dimensional vector spaces $\mathcal{V}_{i}$ over $F$, and a subset $\mathfrak{Z}$ in $\prod \mathcal{V}_{i}^{0}$ such that the image of $\mathfrak{Z}$ under the $i$-th projection does not contain zero in its closure, the closure of $\mathfrak{Z}$ in $\prod \tilde{\mathbb{P}}\left(\mathcal{V}_{i}\right)^{0}$ is compact (where we used notations from 2.5.2); hence for any neighborhood $V$ of $\prod \mathbb{P}\left(\mathcal{V}_{i}\right)$, multiplication by $\pi^{-N}$ sends $\mathfrak{Z}$ to $V$ for some $N$.

To prove (b) it is enough to show the same statement for a $U_{B} \times U_{B}$ orbit. The image of such an orbit in $\operatorname{End}(M)_{i}$ is also a $U_{B} \times U_{B}$ orbit different from $\{0\}$. Since an orbit of a unipotent group on an affine algebraic variety is Zariski closed by Kostant-Rosenlicht Theorem, we get the statement.

Remark 9.5. The implication in part (a) of the lemma is actually an "if and only if" statement, we only proved the direction we need to save space.

The last auxiliary fact needed in the proof of Theorem 7.6 is the following property of intertwining operators which is immediate from its definition.

For $w_{1}, w_{2} \in W$ we let $r\left(\mathcal{M}_{b}^{v}(Y)\right)_{w_{1}, w_{2}}$ denote the corresponding subquotient of the filtration on $r\left(\mathcal{M}_{b}^{v}(Y)\right)$ induced by the stratification of $Y$ by $B \times B$ orbits. 
Claim 9.6. The component $r\left(\mathcal{S}_{b}(X)\right)_{w_{1}, w_{2}} \rightarrow r\left(\mathcal{M}_{b}^{v}(Y)\right)_{w_{1}^{\prime}, w_{2}^{\prime}}$ of $I_{v}$ equals identity if $w_{1}^{\prime}=v w_{1}, w_{2}^{\prime}=w_{2} w_{0} v^{-1}$ and $\ell\left(w_{1}\right)=\ell(v)+\ell\left(w_{1}^{\prime}\right), \ell\left(w_{2}\right)=\ell\left(w_{2}^{\prime}\right)+\ell\left(w_{0} v^{-1}\right)$.

9.2. Proof of Theorem [7.6. Comparing Proposition 7.1 with Corollary 7.4 we see that the composition $I_{w} \mathrm{~B}^{\star}$ is well defined.

Then Lemma 9.3 together with Claim 9.6 show that $r\left(I_{w} B^{\star}\right)$ induces a quotient map $r(\mathcal{S}(G))_{1} \rightarrow r\left(\mathcal{M}_{b}^{w}(Y)\right)_{1,1}$ which is equal to the canonical embedding $\mathcal{S}(T) \rightarrow$ $\mathcal{S}_{b}^{w}(T)$. By Frobenius adjointness this implies that $I_{w} B^{\star}$ is the composition of $\mathrm{A}$ with the embedding $\mathcal{M}(Y) \rightarrow \mathcal{M}_{b}^{w}(Y)$.

\section{Plancherel type formulas}

In this last section we present an application of Corollary 7.8

10.1. The map $B$ and the Plancherel functional. The equality $B=A^{\star}\left(I_{w}^{\star}\right)^{-1}$ allows one to write down a spectral expression for the value $f(1)$ for a function $f \in \mathcal{S}(G)$ presented in the form $f=\mathrm{B}(\phi), \phi \in \mathcal{S}(X)$. To spell this out we need some preliminaries.

Let Char denote the space of characters of the torus $\mathbb{T}$ viewed as an affine ind-algebraic variety over $\mathbb{C}$. We have Char $\cong \bigsqcup_{\chi \in \mathbb{T}(O)^{\vee}} C_{\text {. }}$. War ${ }_{\chi}$, where $\mathbb{T}(O)^{\vee}$ is the discrete set of characters of $\mathbb{T}(O)$ and Char $_{\chi}$ is the space of characters whose restriction to $T(O)$ is equal to $\chi$. Then $C h a r_{\chi}$ is a principal homogeneous space for the dual torus $\mathbb{T}^{\vee}$ over $\mathbb{C}$. Let $\mathcal{O}^{f}($ Char $)=\bigoplus_{\chi} \mathcal{O}\left(\right.$ Char $\left._{\chi}\right)$ denote the (nonunital) ring of regular functions on Char which vanish on all but a finite number of components. We have a canonical isomorphism $\mathcal{O}^{f}(C h a r) \cong \mathcal{S}(\mathbb{T})$, and a noncanonical isomorphism $\mathcal{O}^{f}($ Char $) \cong \bigoplus_{\chi \in \mathbb{T}(O)^{\vee}} \mathcal{O}\left(\mathbb{T}^{\vee}\right)$.

Recall the partial compactification $\overline{\mathbb{T}}_{\text {aff }}$ of the torus $\mathbb{T}$ given by

$$
\overline{\mathbb{T}}=\operatorname{Spec}\left(F\left[X^{*}(\mathbb{T})^{+}\right)\right)
$$

where $X^{*}(\mathbb{T})^{+} \subset X^{*}(\mathbb{T})$ is the semigroup consisting of positive rational combinations of simple roots in $X^{*}(\mathbb{T})$. Let $\mathcal{S}_{b}(\mathbb{T})$ be the space of distributions on $\overline{\mathbb{T}}_{\text {aff }}$ invariant with respect to an open subgroup in $\mathbb{T}$ whose support is contained in a compact subset of $\overline{\mathbb{T}}_{\text {aff. }}$. To give a spectral description of this ring introduce the affine toric variety $\overline{\mathbb{T}}^{\vee}=\operatorname{Spec}\left(\mathbb{C}\left[X_{*}(\mathbb{T})^{+}\right]\right)$, where $X_{*}(\mathbb{T})^{+}$is the dual cone to $X^{*}(\mathbb{T})^{+}$, i.e., $X^{*}(\mathbb{T})^{+} \subset X_{*}(\mathbb{T})=X^{*}\left(\mathbb{T}^{\vee}\right)$ is the set of weights which are positive rational linear combinations of dominant weights of $\mathbb{T}^{\vee}$. This defines a partial compactification $\overline{C h a r}_{\chi}=\left(\overline{\mathbb{T}} \times C h a r_{\chi}\right) / \mathbb{T}$ of the principal homogeneous space $C h a r_{\chi}$. Let $\widehat{\mathcal{O}}\left(C h a r_{\chi}\right)$ be the ring of functions on the punctured formal neighborhood of the divisor $\partial \operatorname{Char}_{\chi}=\overline{C h a r}_{\chi} \backslash C h a r_{\chi}$. Set $\widehat{\mathcal{O}}(\text { Char })^{f}=\bigoplus_{\chi} \widehat{\mathcal{O}}\left(\right.$ Char $\left._{\chi}\right)$. Then it is easy to see that $\widehat{\mathcal{O}}(\text { Char })^{f} \cong \mathcal{S}_{b}(\mathbb{T})$. Choosing a point in $C h a r_{\chi}$ we get identifications $\operatorname{Char}_{\chi} \cong \mathbb{T}^{\vee}, \overline{C h a r}_{\chi} \cong \overline{\mathbb{T}}^{\vee}$,

$$
\widehat{\mathcal{O}}_{\chi} \cong\left\{\phi: X_{*}(\mathbb{T}) \rightarrow \mathbb{C} \mid \operatorname{supp}(\phi) \subset S+X_{*}(\mathbb{T})^{+} \text {for } S \subset X_{*}(\mathbb{T}),|S|<\infty\right\} .
$$

We define a linear functional $\int: \widehat{\mathcal{O}}_{\chi} \rightarrow \mathbb{C}$ sending a function $\phi: X_{*}(\mathbb{T}) \rightarrow \mathbb{C}$ to $\phi(0)$. It is easy to see that this is independent of the choice of a point in $C_{\text {a }} r_{\chi}$. If $f \in \widehat{\mathcal{O}}\left(\operatorname{Char}_{\chi}\right)$ is a Laurent expansion of a rational function on Char $_{\chi}$ (we assume that the divisor of poles of $f$ does not pass through the zero-dimensional orbit $\left\{t_{0}^{\chi}\right\}$ of $\mathbb{T}^{\vee}$ on $\overline{C h a r}_{\chi}$, so that the Laurent expansion is well defined), then $\int f$ is the integral of $f$ over a coset of the maximal compact subtorus close to $t_{0}^{\chi}$, hence the 
notation. We let $\int: \widehat{\mathcal{O}}(\text { Char })^{f} \rightarrow \mathbb{C}$ be the linear functional coinciding with the above functional on $C h a r_{\chi}$ for every $\chi$. It is easy to see that under the isomorphism $\widehat{\mathcal{O}}(\text { Char })^{f} \cong \mathcal{S}_{b}(\mathbb{T})$, the functional $\int$ goes to the functional $h \mapsto h(1)$.

For a character $\chi$ of $T$ let $i_{\chi}: \mathcal{H} \rightarrow \operatorname{End}\left(V_{\chi}\right), i_{\chi}^{-}: \mathcal{H} \rightarrow \operatorname{End}\left(V_{\chi}^{-}\right)$denote the induced representations $i_{B}^{G}(\chi), i_{B}^{-G}(\chi)$ respectively.

Recall from subsection 6.1 that elements in $\mathcal{M}(Y)$ can be interpreted as operators acting on $\mathcal{S}(G / U)$ which commute with the action of the abstract Cartan $\mathbb{T}$ and are invariant with respect to an open compact subgroup, or as family of operators acting on the space $V_{\chi}$ for every $\chi$. In particular, for $f \in \mathcal{M}(Y)$ we get a regular function $\tau(f) \in \mathcal{O}^{f}($ Char $)$ whose value at $\chi$ equals $\operatorname{Tr}\left(f, V_{\chi}\right)$.

It is easy to see that $\left(\mathcal{M}_{b}^{1}\right)_{s m}(Y)=\widehat{\mathcal{O}}($ Char $) \otimes_{\mathcal{O}(\text { Char })} \mathcal{M}(Y)$, where $\left(\mathcal{M}_{b}^{1}\right)_{s m} \subset$ $\mathcal{M}_{b}^{1}$ denotes the subspace of elements invariant under some open subgroup in $G \times G$. Thus for $f \in\left(\mathcal{M}_{b}^{1}\right)_{s m}(Y)$ we get $\tau(f) \in \widehat{\mathcal{O}}($ Char $)$.

In the next statement we use Proposition 7.5.

Claim 10.1. a) For $f \in\left(\mathcal{M}_{b}^{1}\right)_{s m}(Y)$ we have

$$
\int \tau(f)=\int_{\Delta_{\mathcal{B}} \times\{1\}} f=\mathrm{A}^{\star}(f)(1),
$$

where $\Delta_{\mathcal{B} \times\{1\}} \subset \operatorname{pr}_{Y}^{-1}\left(\mathcal{B}^{2}\right) \subset Y$ is defined by means of (2.2) 4

b) For any $\phi \in \mathcal{S}(X)$ we have

$$
\mathrm{B}(\phi)(1)=\int \tau\left(\left(I_{1}^{\star}\right)^{-1}(\phi)\right) .
$$

Proof. Pick an open compact subgroup $K \subset G$ such that $f \in\left(\mathcal{M}_{b}^{1}\right)^{K \times K}(Y)$. The $\mathcal{O}($ Char $)$-module $\mathcal{S}(G / U)^{K}$ splits as a sum where each summand is free over the ring functions on a component of Char; moreover, each module has a basis indexed by the finite set $K \backslash G / B$. For $f \in \mathcal{M}(Y)$ the sum of diagonal entries of the corresponding operator on $\mathcal{S}(G / U)$ in this basis is readily seen to equal the element

$$
\operatorname{pr}_{2 *}\left(\left.f\right|_{p r_{Y}^{-1}}\left(\Delta_{\mathcal{B}}\right)\right) \in \mathcal{S}(\mathbb{T}) \cong \mathcal{O}^{f}(\text { Char }),
$$

where $p r_{2}$ denotes projection to the second factor in (2.2) and we use the observation in footnote 4 to make sense of $p r_{2 *}$. Applying the functional $\int: \mathcal{O}^{f}($ Char $) \rightarrow \mathbb{C}$ to this sum of diagonal elements yields $\int \tau(f)$, which is thus seen to be equal to the second expression in (10.1). This proves the first equality for $f \in \mathcal{M}(Y)$, the case of $f \in\left(\mathcal{M}_{b}^{1}\right)_{s m}(Y)$ follows. The second equality in (a) is clear since the preimage of $1 \in G$ under the projection $(G \times G) / B \rightarrow G$ maps isomorphically to $\mathcal{B} \times\{1\} \subset \operatorname{pr}_{Y}^{-1}\left(\Delta_{\mathcal{B}}\right) \subset Y$.

This proves part (a); part (b) follows from (a) compared to Corollary 7.8,

Remark 10.2. A similar equality holds where $I_{1}$ is replaced by $I_{w}$ for some $w \in W$.

10.2. Commutative subalgebras in the Hecke algebra. We fix a maximal split torus $T \subset G$ and a subgroup $K \subset K_{0} \subset G$ (where $K_{0}$ is as in subsection 5.3) which is nice in the sense of [2] and is in a good relative position to $T$. Recall that this means that

$$
K=K^{+} \cdot K^{0} \cdot K^{-}
$$

\footnotetext{
${ }^{4}$ Notice that for $f \in\left(\mathcal{M}_{b}^{1}\right)_{s m}(Y)$ the restriction $\left.f\right|_{\Delta_{\mathcal{B}} \times\{t\}}, t \in \mathbb{T}$ is a locally constant measure on $\mathcal{B}$, thus the second integral in the displayed formula is well defined.
} 
for any pair of opposite Borel subgroups $B^{+}=U^{+} T, B^{-}=U^{-} T$, where $K^{+}=$ $U^{+} \cap K, K^{-}=U^{-} \cap K, K^{0}=T \cap K$. We fix a Borel subgroup containing $T$, this defines the cone of dominant coweights $X_{*}(T)^{+} \subset X_{*}(T)$.

The following technical statement is not hard but it plays an important role in Bernstein's theory.

Recall notation $\iota, \iota_{I}$ introduced in subsection 5.3. For $\nu \in X_{*}(T)^{+}$let $e_{\nu} \in \mathcal{H}$ be the delta-function of the two-sided coset $K \iota(\nu) K$.

Let $x_{\nu} \in \mathcal{S}(X)$ be the delta-function of the $K \times K$ orbit of $\iota_{X}(\nu)$. We set $\theta_{\nu}=\mathrm{B}\left(x_{\nu}\right)$.

Proposition 10.3. The map $X_{*}(T) \rightarrow \mathcal{H}, \nu \mapsto \theta_{\nu}$ is uniquely characterized by the following two properties.

i) $\theta_{\mu_{1}+\mu_{2}}=\theta_{\mu_{1}} \theta_{\mu_{2}}$.

ii) There exists $\nu_{0}$ such that for $\nu \in \nu_{0}+X_{*}(T)^{+}$, we have $\theta_{\nu}=e_{\nu}$.

We will need the following lemma.

Lemma 10.4. 2] a) For $\mu, \nu \in X_{*}(T)^{+}$we have $e_{\mu} e_{\nu}=e_{\mu+\nu}$.

b) Given $\lambda \in X_{*}(T)^{+}$, there exists $N>0$ such that the kernel of left multiplication by $e_{N \lambda}$ on $\mathcal{H}_{K}$ equals the kernel of left multiplication by $e_{(N+1) \lambda}$.

Proof. (a) follows directly from (10.2). (b) follows from the fact that $\mathcal{H}_{K}$ is right Noetherian.

Proof of the Proposition. To check uniqueness, assume $\theta_{\mu}$ and $\theta_{\mu}^{\prime}$ are different collections of elements satisfying (i), (ii). Fix $\nu \in X_{*}(T)^{+}$such that $\theta_{\nu}=e_{\nu}=\theta_{\nu}^{\prime}$. We have also $\theta_{\mu+N \nu}=e_{\mu+N \nu}=\theta_{\mu+N \nu}^{\prime}$ for some $N$. Thus $\theta_{\mu}-\theta_{\mu}^{\prime}$ lies in the image of left multiplication by $e_{\nu}$, as well as in the kernel of left multiplication by $e_{N \nu}$. Lemma 10.4(b) implies that $\theta_{\mu}=\theta_{\mu}^{\prime}$, which proves uniqueness.

It remains to check that $\theta_{\nu}=\mathrm{B}\left(x_{\nu}\right)$ does satisfy (i), (ii). It follows from Lemma 5.5 (b) that given an admissible bijection between $U \subset G$ and $V \subset X$ there exists $\nu_{0} \in X_{*}(T)^{+}$such that the bijection sends the set $K \iota_{G}(\nu) K$ into the set $K \iota_{X}(\nu) K$ for $\nu \in \nu_{0}+X_{*}(T)^{+}$. Thus (ii) follows from the construction of B. Given $\nu \in$ $X_{*}(T)^{+}$, Proposition 3.13 implies that there exists $\nu_{0} \in X_{*}(T)^{+}$such that

$$
e_{\nu} * x_{\mu}=x_{\nu+\mu}=x_{\mu} * e_{\nu}
$$

for $\mu \in \nu_{0}+X_{*}(T)^{+}$. Since the elements $x_{\mu}$ are permuted by the action of $\mathbb{T}$ commuting with the action of $\mathcal{H} \otimes \mathcal{H}$, we see that (10.3) holds for all $\mu$. Hence $e_{\nu} \theta_{\mu}=\theta_{\nu+\mu}$ for all $\mu$. We now proceed to check (i). Pick $\nu$ such that $e_{\eta}=\theta_{\eta}$ when $\eta$ is either $\nu+\mu_{1}$, or $\nu+\mu_{1}+\mu_{2}$. Then we see that $e_{\nu} \theta_{\mu_{1}+\mu_{2}}=e_{\nu+\mu_{1}+\mu_{2}}=e_{\nu} \theta_{\mu_{1}} \theta_{\mu_{2}}$. Also, both $\theta_{\mu_{1}+\mu_{2}}$ and $\theta_{\mu_{1}}, \theta_{\mu_{2}}$ lie in the image of left multiplication by $e_{N \nu}$ for all $N$. Thus the desired equality follows from Lemma 10.4(b).

Example 10.5. The case most often encountered in the literature is when $K=I$ is the Iwahori subgroup. Then $\mathcal{H}=\mathcal{H}_{I}$ is isomorphic to the affine Hecke algebra, it contains invertible elements $\theta_{\nu}^{I}, \nu \in \Lambda$ such that $\theta_{\nu_{1}+\nu_{2}}^{I}=\theta_{\nu_{1}} \cdot \theta_{\nu_{2}}^{I}$ and $\theta_{\nu}^{I}=\delta_{I \iota(\nu) I}$ when $\nu$ is dominant; they form a part of a system of generators for this algebra discovered by Bernstein [13, which have found numerous important applications. It is clear that these are exactly the elements described in Proposition 10.3 in the case $K=I$. 
We point out a special case of this potentially useful in applications. Setting $\nu=0$ we get

$$
\mathrm{B}\left(\delta_{I_{X}(0) I}\right)=\theta_{0}^{I}=\delta_{I} .
$$

Furthermore, applying averaging with respect to $K_{0} \times K_{0}$ to both sides and using that $X(O)=K_{0} \cdot \iota_{X}(0) \cdot K_{0}$ we get

$$
\mathrm{B}\left(\delta_{X(O)}\right)=\delta_{K_{0}} .
$$

10.3. Proof of Proposition 7.2, It is easy to see that

$$
X \cap \bar{X}_{\text {aff }}(O)=\bigcup_{\lambda \preceq \mathbb{Q} 0} X_{\lambda},
$$

where we write $\lambda \preceq_{\mathbb{Q}} \mu$ if $\mu-\lambda$ is a linear combination of positive coroots with nonnegative rational coefficients.

Using the notation and the content of Example 10.5 we reduce the claim to showing that

$$
\operatorname{supp}\left(\theta_{\lambda}^{I}\right) \cap K_{0}=\emptyset \quad \text { unless } \quad \lambda \preceq \mathbb{Q} 0 .
$$

In fact, we will show that

$$
\operatorname{supp}\left(\theta_{\lambda}^{I}\right) \cap K_{0}=\emptyset \quad \text { unless } \quad \lambda \preceq 0,
$$

where $\preceq$ is standard order on weights, i.e. $\lambda \preceq \mu$ if $\mu-\lambda$ is a sum of positive roots.

We have the standard basis $t_{w}$ of $\mathcal{H}_{I}, w \in W_{\text {aff }}$ where $W_{\text {aff }}$ is the extended affine Weyl group. We also have an algebra anti-involution $i: \mathcal{H}_{I} \rightarrow \mathcal{H}_{I}$ sending $t_{w}$ to $t_{w}^{-1}$ and a pairing $\mathcal{H}_{I} \times \mathcal{H}_{I} \rightarrow k$ given by $\langle f, g\rangle=c_{1}$ where $i(f) g=\sum c_{w} t_{w}$. Then (10.4) reduces to

$$
\left\langle t_{w}, \theta_{\lambda}^{I}\right\rangle=0 \quad \text { for } \quad w \in W, \quad \lambda \npreceq 0,
$$

where $W$ is the finite Weyl group.

Pick a dominant weight $\mu$ such that $\mu+\lambda$ is also dominant. Then for $w \in W$ we have

$$
\left\langle t_{w}, \theta_{\lambda}^{I}\right\rangle=\left\langle t_{\mu} t_{w}, t_{\mu} \theta_{\lambda}\right\rangle=\left\langle t_{(\mu) \cdot w}, \theta_{\mu+\lambda}^{I}\right\rangle .
$$

It is easy to see that for $w, v \in W_{\text {aff }}$ we have $\left\langle t_{w}, t_{v}\right\rangle=0$ unless $v \leq w$ where $\leq$ refers to the Bruhat order on $W_{\text {aff }}$.

For dominant coweights $\eta, \nu$ the inequality $\eta \leq(\nu) w, w \in W$ implies that $\eta \preceq \nu$. So $\left\langle t_{w}, \theta_{\lambda}^{I}\right\rangle \neq 0$ implies that $\mu+\lambda \preceq \mu$, i.e. $\lambda \preceq 0$, which proves (10.5) and hence Proposition 7.2 for adjoint groups.

10.4. Plancherel functional on the abelian subalgebra. Corollary 7.8 shows that

$$
\theta_{\nu}(g)=\mathrm{B}\left(x_{\nu}\right)(g)=\mathrm{A}^{\star} I_{1}(g) .
$$

This yields a spectral expression for the Plancherel functional $f \mapsto f(1)$ restricted to the subalgebra $A_{K} \subset \mathcal{H}$ spanned by $\theta_{\nu}$. To state it we introduce the following notation.

We define a rank one operator $\Pi: V_{\chi}^{K} \rightarrow\left(V_{\chi}^{-}\right)^{K}$ as follows. Notice that the space $V_{\chi}^{K}$ splits as a direct sum of one-dimensional subspaces indexed by the set $K \backslash G / B$; likewise, $V_{\chi}^{-}$is a direct sum of one-dimensional subspaces indexed by $K \backslash G / B^{-}$. The one-dimensional summands corresponding to the coset of 1 in the two spaces are canonically isomorphic (both are identified with the space of the one-dimensional representation $\chi$ of $T$ ), let us denote this space by $\mathbb{C}_{\chi}$. We define 
$\Pi:\left(V_{\chi}^{-}\right)^{K} \rightarrow V_{\chi}^{K}$ as the composition $\left(V_{\chi}^{-}\right)^{K} \rightarrow \mathbb{C}_{\chi} \rightarrow V_{\chi}^{K}$, where the first arrow is the projection arising from the above splitting into a direct sum, and the second arrow is the embedding.

In the next theorem we consider a function $\chi \mapsto \operatorname{Tr}\left(h \Pi R^{-1}, V_{\chi}\right), h \in \mathcal{H}$, where $R: V_{\chi}^{-} \rightarrow V_{\chi}$ is the intertwining operator defined for $\chi$ in a Zariski open dense subset of Char. This is a rational function on Char vanishing on all but a finite number of components. Moreover, in view of Proposition 8.1 for every $\chi$ the point $t_{0} \in \overline{C h a r}_{\chi}$ does not lie in the divisor of poles of this function. Thus the functional $\int$ introduced in subsection 10.1 is well defined on such a function.

Theorem 10.6. For $h \in A_{K}$ we have

$$
h(1)=\int \operatorname{Tr}\left(h \Pi R^{-1}, V_{\chi}^{K}\right) .
$$

Proof. We can assume that $h=\theta_{\nu}$. Applying Corollary 7.8 with $w=1$, we get $\theta_{\nu}=\mathrm{A}^{*} I^{-1}\left(x_{\nu}\right)$.

A function $\phi \in \mathcal{M}_{b}^{w=1}(Y)$ defines an operator on the completion of the universal principal series, $\mathcal{S}(G / U) \otimes_{\mathcal{O}(\text { Char })} \widehat{\mathcal{O}}($ Char $)$.

By Claim 10.1(a) we have

$$
\int \operatorname{Tr}\left(\phi, V_{\chi}\right)=A^{*}(\phi)(1)
$$

where integral in the left-hand side has the same meaning as in the statement of the theorem.

Thus the proof will be complete once we show that

$$
i_{\chi}\left(I_{1}\left(x_{\nu}\right)\right)=i_{\chi}\left(\theta_{\nu}\right) \Pi R^{-1} .
$$

A function $f \in \mathcal{S}(X)$ defines an operator between the principal series and the opposite principal series, $\sigma_{\chi}(f): V_{\chi}^{-} \rightarrow V_{\chi}$. In particular, it is easy to see that $\sigma_{\chi}\left(x_{0}\right)=\Pi$ for all $\chi$. Also, it follows from the definitions that $i_{\chi}\left(\left(I_{1}^{\star}\right)^{-1}(f)\right)=$ $R^{-1} \circ \sigma_{\chi}(f)^{-1}$. Finally, using that all the maps involved commute with the $\mathcal{H} \otimes \mathcal{H}$ action, we get

$$
i_{\chi}\left(\left(I_{1}^{\star}\right)^{-1}\left(x_{\nu}\right)\right)=i_{\chi}\left(\theta_{\nu} * x_{0}\right)=i_{\chi}\left(\theta_{\nu}\right) \Pi R^{-1} .
$$

Thus we have proven (10.7), and hence the theorem.

Remark 10.7. In the special case when $K=I$ is the Iwahori subgroup Theorem 10.6 reduces to (an equivalent form) of [15, Theorem 1.14].

Remark 10.8. We expect that a version of Claim 10.1(b) holds for the space of rapidly decreasing functions on $X$ and that it can be used to deduce the standard Plancherel formula. We plan to develop this application in the future.

\section{APPENDIX: QUASI-NORMAL CONE FOR TORIC COVERINGS}

by Yakov Varshavsky, Roman Bezrukavnikov, and David Kazhdan

In this section we introduce a version of the definition of a normal cone which behaves well for a class of singular varieties, including De Concini-Procesi compactifications of not necessarily adjoint groups. 
11.1. Quasi-normal cone. Let $X$ be a smooth variety over a field $F$ and $Z \subset X$ be a smooth locally closed subvariety. Let $X^{\prime}$ be a normal scheme and $X^{\prime} \rightarrow X$ be a finite flat morphism, let $Z^{\prime}$ be the preimage of $Z$ equipped with reduced subscheme structure. Let $N_{X}(Z)$ be the normal bundle.

Let $\widetilde{N}_{X}(Z)$ be the deformation to the normal cone; thus $\left(\mathbb{A}^{1} \backslash\{0\}\right) \times X \subset$ $\tilde{N}_{X}(Z) \supset N_{X}(Z)$. Recall that $\widetilde{N}$ comes equipped with a $\mathbb{G}_{m}$ action which dilates the fibers of the normal bundle and acts on $\left(\mathbb{A}^{1} \backslash\{0\}\right) \times X$ by $t:(z, x) \mapsto(t z, x)$.

Let $\widetilde{N}_{X^{\prime}}\left(Z^{\prime}\right)$ be the normalization of $\widetilde{N}_{X}(Z)$ in $\left(\mathbb{A}^{1} \backslash\{0\}\right) \times X^{\prime}$, and set $N_{X^{\prime}}\left(Z^{\prime}\right)=$ $\widetilde{N}_{X^{\prime}}(Z) \times_{\mathbb{A}^{1}}\{0\}$. We call $N_{X^{\prime}} Z^{\prime}$ the quasi-normal cone of $Z^{\prime}$ in $X^{\prime}$.

We have a locally closed embedding $Z^{\prime} \times \mathbb{A}^{1} \rightarrow \widetilde{N}_{X^{\prime}} Z^{\prime}$.

Definition. We say that $X^{\prime}$ is well approximated around $Z$ if the following conditions hold.

(a) For some $d>0$ the composition of the natural action of $\mathbb{G}_{m}$ on $\tilde{N}_{Z}(X)$ with the map $\mathbb{G}_{m} \rightarrow \mathbb{G}_{m}, t \mapsto t^{d}$, lifts to an action $\alpha_{d}$ of $\mathbb{G}_{m}$ on $\widetilde{N}_{Z^{\prime}}\left(X^{\prime}\right)$.

(b) Zariski locally on $Z$ there exists an isomorphism $\tau$ between the formal neighborhoods of $Z^{\prime} \times \mathbb{A}^{1}$ in $N_{X^{\prime}}\left(Z^{\prime}\right) \times \mathbb{A}^{1}$ and in $\widetilde{N}_{Z^{\prime}}\left(X^{\prime}\right)$, such that:

(i) $\tau$ restricted to the preimage of $0 \in \mathbb{A}^{1}$ equals identity.

(ii) $\tau$ is $\mathbb{G}_{m}$-equivariant where $\mathbb{G}_{m}$ acts on $\widetilde{N}_{Z^{\prime}}\left(X^{\prime}\right)$ via $\alpha_{d}$ and on $N_{X^{\prime}}\left(Z^{\prime}\right)$ $\times \mathbb{A}^{1}$ by $t:(x, z) \mapsto\left(\alpha_{d}(x), t^{d} z\right)$.

If $F$ is a local field, we can repeat the definition replacing an isomorphism of formal neighborhoods by an analytic isomorphism of actual $F^{\times}$invariant neighborhoods in the space of $F$-points; "Zariski local" should then be replaced by local in the sense of $F$-topology. We will use the term "analytically well approximated" for this version of the property. If $F$ is non-Archimedian, existence of local isomorphisms implies existence of a global isomorphism of appropriate neighborhoods.

In the latter case, the restriction of $\tau$ to the fiber over $1 \in \mathbb{A}^{1}$ will be called an admissible bijection.

Example 11.1. If $X^{\prime}=X$, then $\tau$ amounts to an isomorphism between a neighborhood of $Z$ in $X$ and a neighborhood of the zero section in the normal bundle $N_{X}(Z)$, whose differential in the normal direction equals identity.

11.2. Toric covering. Again let $X$ be a smooth variety over $F$, and let $D_{i}, i \in I$ be smooth divisors with normal crossing in $X$. For a subset $J \subset I$ we have the corresponding stratum $X_{J}=\bigcap D_{i}, i \in J$. We fix $J \subset I$ and let $Z=X_{J}$.

We have line bundles $\mathcal{L}_{i}=\mathcal{O}\left(D_{i}\right)$ on $X$, each coming with a canonical section $s_{i}$. They can be combined into a $T$-bundle $\mathcal{E}$ for the torus $T=\left(\mathbb{G}_{m}\right)^{I}$.

Let $T^{\prime}$ be another torus and $T^{\prime} \rightarrow T$ be a fixed isogeny. Suppose that the above $T$-bundle on $X$ lifts to a $T^{\prime}$-bundle $\mathcal{E}^{\prime}$ (i.e. $\mathcal{E}$ is the push-forward of $\mathcal{E}^{\prime}$ ); we fix such a $T^{\prime}$-bundle $\mathcal{E}^{\prime}$. This data defines a ramified covering $X^{\prime} \rightarrow X$ as follows.

Let $A=\left(\mathbb{A}^{1}\right)^{I}$, thus $T$ acts on $A$ making it a toric variety. The $T$-bundle $\mathcal{E}$ defines an associated bundle $A_{\mathcal{E}}$ with the fiber $A$; of course $A_{\mathcal{E}}$ is nothing but the total space of the sum of line bundles $\mathcal{L}_{i}$; the sections $s_{i}$ combine to a section $s: X \rightarrow A_{\mathcal{E}}$.

Let $A^{\prime}$ be the normalization of $A$ in the covering $T^{\prime} \rightarrow T$. We can also form an associated bundle $A_{\mathcal{E}^{\prime}}^{\prime}$. Set $X^{\prime}=X \times_{A_{\mathcal{E}}} A_{\mathcal{E}^{\prime}}^{\prime}$. We call $X^{\prime}$ obtained by this construction a toric covering. 
For a subset $J \subset I$ we let $A^{\prime}(J)$ be the normalization of $\left(\mathbb{A}^{1}\right)^{J}$ in the quotient of $T^{\prime}$ by the neutral connected component of the preimage of $\left(\mathbb{G}_{m}\right)^{I \backslash J} \subset T$, let $A_{\mathcal{E}^{\prime}}^{\prime}(J)$ be the corresponding bundle over $X$.

Example 11.2. Let $G$ be a reductive algebraic group. Let $G_{a d}=G / Z(G)$, where $Z(G) \subset G$ is the center, and let $G^{\prime} \subset G$ be the derived (commutator) subgroup. Let $X=\left(G / G^{\prime}\right) \times \bar{G}_{a d}$ where $\bar{G}_{a d}$ is the wonderful compactification of $G_{a d}$, and $D_{i}$ be the components of the complement $X \backslash\left(G / G^{\prime}\right) \times G_{a d}$. The torus $T$ is identified with the (abstract) Cartan subgroup of $G_{a d}$. The total space of $\mathcal{E}$ is easily seen to be identified with $\left(G / G^{\prime}\right) \times S^{0}\left(G_{a d}\right)$, where $S^{0}\left(G_{a d}\right) \subset S\left(G_{a d}\right)$ is an open part in Vinberg's semi-group $S\left(G_{a d}\right)$. Let $T^{\prime}$ be the abstract Cartan of $G^{\prime}$, and let $\mathcal{E}$ be the $T$-bundle whose total space is $S^{0}(G)$. (If $G$ is simply-connected, this is a universal torus bundle over $X$, i.e., the map from $X^{*}(T)$ to $\operatorname{Pic}(X)$ given by this bundle is an isomorphism; we neither prove nor use this fact.) It is easy to see that the preimage of $\left(G / G^{\prime}\right) \times G_{a d} \subset X$ in $X^{\prime}$ is isomorphic to $G$. It follows from the theorem below that $X^{\prime}$ is the normalization of $\left(G / G^{\prime}\right) \times \bar{G}_{a d}$ in $G$.

Example 11.3. Assume that the line bundles $\mathcal{L}_{i}$ are trivial (i.e. $D_{i}$ is cut out by a global function). Fix $\mathcal{E}^{\prime}$ to be the trivial $T^{\prime}$-torsor. Then $s$ amounts to a smooth morphism $X \rightarrow A$, so that $Z$ is the preimage of the closure of a $T$-orbit in $A$; we have $X^{\prime}=X \times_{A} A^{\prime}$.

11.3. Theorem. Let $X^{\prime} \rightarrow X \supset D_{i}$ be a toric covering and $Z=X_{J}$ as above.

a) $X^{\prime}$ is a normal variety and the map $X^{\prime} \rightarrow X$ is finite and flat.

b) $X^{\prime}$ is well approximated around $Z$. If $F$ is a local field it is also analytically well approximated.

c) The quasi-normal cone $N_{X^{\prime}}\left(Z^{\prime}\right)$ is canonically isomorphic to $A_{\mathcal{E}^{\prime}}^{\prime}(J) \times_{X} Z$.

Remark. If $T^{\prime}=T$, so that $X^{\prime}=X$, the isomorphism of part (c) amounts to the adjunction formula $\left.\mathcal{O}(D)\right|_{D} \cong N_{X}(D)$.

Proof. It is easy to check that all the definitions above are compatible with smooth base change. Thus it suffices to check that the statements hold after base change with respect to the morphism from the total space of the torsor $\mathcal{E}$ to $X$. This reduces the proof to the situation of Example 11.3. Using compatibility with smooth base change again, we see that it suffices to show that $A^{\prime}$ is normal, $A^{\prime} \rightarrow A$ is flat and finite; that $A^{\prime}$ is well approximated around the closure $A_{J}$ of a $T$-orbit in $A$, and that $N_{A^{\prime}}\left(A_{J}^{\prime}\right)=A^{\prime}$. Normality and finiteness are clear from the definition, and flatness is easy to show. The deformation to the normal cone $\widetilde{N}_{A}\left(A_{J}\right)$ is constant, which implies the rest.

Remark. Part (c) of the theorem shows that an open part of $N_{X^{\prime}}\left(Z^{\prime}\right)$ is identified with the total space of $\left.\mathcal{E}^{\prime}\right|_{Z}$. In the situation of Example 11.2, this is the space denoted by $X_{J}$ in this paper.

\section{ACKNOWLEDGEMENTS}

The authors are grateful to Joseph Bernstein for many discussions and explanations over the years. We also thank Michael Finkelberg and Yakov Varshavsky for some related discussions and Vladimir Drinfeld and Jonathan Wang for helpful comments on the text. 


\section{REFERENCES}

[1] J. Bernstein, unpublished notes, available at Drinfeld's seminar webpage.

[2] J. N. Bernstein, Le "centre" de Bernstein (French), Representations of reductive groups over a local field, Travaux en Cours, Hermann, Paris, 1984, pp. 1-32. Edited by P. Deligne. MR771671 (86e:22028)

[3] R. Bezrukavnikov, M. Finkelberg, and V. Ostrik, Character D-modules via Drinfeld center of Harish-Chandra bimodules, Invent. Math. 188 (2012), no. 3, 589-620, DOI 10.1007/s00222011-0354-3. MR 2917178

[4] M. Brion, Lectures on the geometry of flag varieties, http://www-fourier.ujfgrenoble.fr/ mbrion/lecturesrev.pdf.

[5] M. Brion, The behaviour at infinity of the Bruhat decomposition, Comment. Math. Helv. 73 (1998), no. 1, 137-174, DOI 10.1007/s000140050049. MR1610599 (99b:14049)

[6] M. Brion and S. Kumar, Frobenius splitting methods in geometry and representation theory, Progress in Mathematics, vol. 231, Birkhäuser Boston, Inc., Boston, MA, 2005. MR2107324 (2005k:14104)

[7] M. Brion and P. Polo, Large Schubert varieties, Represent. Theory 4 (2000), 97-126 (electronic), DOI 10.1090/S1088-4165-00-00069-8. MR.1789463(2001j:14066)

[8] W. Casselman, Introduction to admissible representations of p-adic groups, preprint available at: https://www.math.ubc.ca/ cass/research/publications.html.

[9] C. De Concini and C. Procesi, Complete symmetric varieties, Invariant theory (Montecatini, 1982), Lecture Notes in Math., vol. 996, Springer, Berlin, 1983, pp. 1-44, DOI 10.1007/BFb0063234. MR718125 (85e:14070)

[10] I. M. Gel'fand, M. I. Graev, and I. I. Pyatetskii-Shapiro, Representation theory and automorphic functions, Generalized Functions, vol. 6, Academic Press, Inc., Boston, MA, 1990. Translated from the Russian by K. A. Hirsch; Reprint of the 1969 edition. MR 1071179 (91g:11052)

[11] S. Evens, B. F. Jones, On the wonderful compactification, arxiv:0801.0456.

[12] X. He and J. F. Thomsen, Geometry of $B \times B$-orbit closures in equivariant embeddings, Adv. Math. 216 (2007), no. 2, 626-646, DOI 10.1016/j.aim.2007.06.001. MR2351372(2008k:14092)

[13] G. Lusztig, Some examples of square integrable representations of semisimple p-adic groups, Trans. Amer. Math. Soc. 277 (1983), no. 2, 623-653, DOI 10.2307/1999228. MR694380 $(84 \mathrm{j}: 22023)$

[14] M. Marten, M. Thaddeus, Compactifications of reductive groups as moduli stacks of bundles, arXiv:1105.4830

[15] E. M. Opdam, A generating function for the trace of the Iwahori-Hecke algebra, Studies in memory of Issai Schur (Chevaleret/Rehovot, 2000), Progr. Math., vol. 210, Birkhäuser Boston, Boston, MA, 2003, pp. 301-323. MR1985730 (2004f:20013)

[16] D. Renard, Représentations des groupes réductifs p-adiques (French), Cours Spécialisés [Specialized Courses], vol. 17, Société Mathématique de France, Paris, 2010. MR2567785 (2011d:22019)

[17] Y. Sakellaridis, A. Venkatesh, Periods and harmonic analysis on spherical varieties, preprint arXiv:1203.0039, 291pp.

[18] T. A. Springer, Some results on compactifications of semisimple groups, International Congress of Mathematicians. Vol. II, Eur. Math. Soc., Zürich, 2006, pp. 1337-1348. MR2275648 (2008a:14063)

[19] T. A. Springer, Linear algebraic groups, 2nd ed., Progress in Mathematics, vol. 9, Birkhäuser Boston, Inc., Boston, MA, 1998. MR1642713 (99h:20075)

(R. Bezrukavnikov) Department of Mathematics, Massachusetts Institute of Technology, Cambridge, Massachusetts 02139 - and - National Research University Higher School of Economics, International Laboratory of Representation Theory and Mathematical Physics, 20 Myasnitskaya st., Moscow 101000, Russia

E-mail address: bezrukav@math.mit.edu

(D. Kazhdan) Department of Mathematics, Hebrew University, Jerusalem, Israel

E-mail address: kazhdan@math.huji.ac.il

(Y. Varshavsky) Department of Mathematics, Hebrew University, Jerusalem, Israel

E-mail address: vyakov@math.huji.ac.il 